\title{
Asynchronous Adaptive Fault-Tolerant Control for Networked Stochastic Unmanned Surface Vehicles with Multiple Types of Actuator Faults
}

\author{
Ai-Min Wang $(\mathbb{D}$, Jian-Ning $L i(\mathbb{D}$, and Xiao-Bin Xu $\mathbb{D}$ \\ Institute of System Science and Control Engineering, School of Automation, Hangzhou Dianzi University, \\ Hangzhou 310018, China \\ Correspondence should be addressed to Jian-Ning Li; ljn@hdu.edu.cn
}

Received 29 July 2020; Revised 27 August 2020; Accepted 20 October 2020; Published 5 November 2020

Academic Editor: Honglei Xu

Copyright ( $\odot 2020$ Ai-Min Wang et al. This is an open access article distributed under the Creative Commons Attribution License, which permits unrestricted use, distribution, and reproduction in any medium, provided the original work is properly cited.

This paper aims to design an asynchronous adaptive fault-tolerant controller for the networked stochastic unmanned surface vehicles (NSUSVs) subject to multiple types of actuator faults and external disturbance. The partial fault and bias fault of the actuator are taken into consideration simultaneously. By estimating online the unknown bias fault of the actuator and the external disturbances, the proposed adaptive fault-tolerant controller can automatically compensate for these impacts produced by actuator faults and external perturbation while preserving the uniformly ultimate boundedness of the solutions. Both the faulty actuator and the designed controller are asynchronous with the NSUSVs. Moreover, a mode-dependent adaptive event-triggered mechanism (AETM) is introduced in order to facilitate network resources utilization. Finally, the effectiveness and correctness of the proposed design scheme are verified by a numerical example.

\section{Introduction}

Happening along with the rapid development and continuous renewal of the AI, Big Data, and the Internet of Things, the marine vehicles trend toward advanced automation. Unmanned marine vehicles (such as unmanned surface vehicles (USVs)) are easier to deploy and operate than manned marine vehicles in some risky and extreme environments, so they have remarkable advantages in field operations, which makes them widely used in civil and military fields (see [1-4] and the references therein). Besides, due to the agile controllability and strong autonomy of USVs, a growing number of scholars have begun to conduct more in-depth research on various control strategies for USVs. Especially, the heading control strategy for USVs has been intensively addressed in $[5,6]$. The maneuvering control method of USVs has been vastly investigated in $[7,8]$. The trajectory tracking control approach has been extensively studied in [9-12].

Note that USVs are usually dynamically located and remotely controlled by the land control station through advanced communication technologies such as Wi-Fi or radio modems. Therefore, USVs, communication network, and onshore control station constitute the networked unmanned surface vehicles (NUSVs). Since the communication network is introduced into USVs, the connectivity of the network might be neither constant nor simply switching between networks in a specific way, but randomly switching. The random switching of NUSVs may be caused by channel fading, packet dropouts, link failures, etc. Therefore, it is proper to model the randomly switching network topologies as a Markov process [13], which has not been taken into consideration in many of the existing works about USVs. The issue of networked stochastic unmanned surface vehicles (NSUSVs) has not yet received research attention and remains to be solved.

Meanwhile, on account of USVs controlled in the network environment, they inevitably lead to communication problems in the proposed NSUSVs, such as time delay, packet loss, and quantization, which will result in the loss of information. In the NSUSVs, incomplete information 
transmission is responsible for the asynchronization phenomenon that the modes of the controller/filter, even the actuator, do not run synchronously with the modes of the original system. This problem has been way settled by many scholars as soon as it was put forward. For instance, the authors of [14-18] have portrayed the asynchronization phenomenon appears between system modes and controller modes as a so-called hidden Markov model (HMM). Analogically, the asynchronization phenomenon exists between system modes, and filter modes have been exhaustively discussed in [19-22]. Detailedly, in [22], an asynchronous filter is proposed for the original system with quantized measurements where the filter and quantizer are both modedependent, and their modes are asynchronous with that of the system, which is described by two corresponding HMMs. However, as far as the author knows, there is still a gap in the research of asynchronous problems among controllers, actuators, and systems. In addition to this problem, it is not difficult to find that the above references all adopt the proposed timetriggered mechanism (TTM), which inevitably leads to the waste of limited network resources. In order to limit the number of packets sent over the network, the event-triggered mechanism (ETM) is applied to HMM in [23, 24], but relevant approaches discussed in $[23,24]$ still have the drawback that the event-triggered threshold is assumed to be a preset fixed value, which makes it difficult to adapt to the changes of the system. Motivated by [25], a novel mode-dependent AETM with the adaptive threshold is designed. This problem is still a challenging and interesting research problem that promotes our current research work.

All the references mentioned hereinabove assumes that the components of the system are running normally. However, in actual control systems, the actuator may suffer from uncertainties [26] or some failures, such as loss of effectiveness, bias, and outage fault, which will degrade the performance of the system or even crash the system. The fault-tolerant control (FTC) approach can automatically compensate for component failures while maintaining system stability. FTC can be classified into active fault-tolerant control (AFTC) and passive faulttolerant control (PFTC). Note that their similarities and differences are elaborated at length in [27] and will not be repeated here because of limited space. The concentration on actuator failure has been increasing in recent decades. For instance, the study on partial failures of actuators has been vastly proposed in [28-34]. The problem regarding actuator bias faults has been discussed in [35-38]. Regrettably, only a single actuator failure model is considered in the aforementioned works about actuator faults. Although the passive fault-tolerant controller is designed in [39] for mixed actuator faults, which include the actuator bias fault and the actuator partial fault, the result is conservatism caused by the application of the PFTC method. However, it is gratifying that the adaptive faulttolerant control approach can solve this kind of hybrid actuator faults [40-42]. As a matter of fact, the adaptive fault-tolerant control approach, as a branch of AFTC, can compensate for the failures well by reconstructing a new controller online by forming a suitable fault-tolerant control law (for details, see $[29,31,33-37,40-43]$ and references therein), which is the second motivation of this paper.

The purpose of this paper is to design an adaptive faulttolerant controller for NSUSVs that suffer from external disturbance and multiple types of actuator faults. First, two HMMs are used to describe the asynchronization among the controller, actuator, and the proposed system. By introducing a mode-dependent AETM, the resources transferred to the network can be limited effectively. An adaptive fault-tolerant control method using the adaptive algorithm is designed to estimate and compensate for the unknown actuator faults. Compared with existing work, the major contributions of our design method can be summarized as follows:

(1) In this work, a unified actuator faults model including the partial faults and the bias faults is taken into consideration. It is worth mentioning that we skillfully couple the unknown external disturbances and the unknown bias faults into the proposed augmented disturbances. Then, corresponding online adaptive protocols are constructed to track and estimate its upper and lower bounds simultaneously.

(2) The asynchronous phenomenon considered in this paper exists not only between the controller and the proposed NSUSVs but also between the actuator and the proposed NSUSVs. In other words, both the controller and the actuator are asynchronous with the controlled NSUSVs, which are described as two HMMs.

(3) To enhance the efficiency of network resources usage, a mode-dependent AETM with the network-induced delay is constructed, where the proposed triggered threshold is designed to be a time-varying variable.

The remainder of this paper is structured as follows. The establishment of the NSUSVs model with actuator faults under a mode-dependent AETM is described in Section 2. Based on the constructed model, the main results are depicted in Section 3. A numerical example is given in Section 4. Eventually, there is a conclusion in Section 5.

Notations in this work are as follows. Given symmetric square matrices $X$ and $Y, X \geq Y$ (respectively, $X \leq Y$ ) means that $X-Y$ is positive semidefinite (respectively, negative semidefinite). Similarly, $X>Y$ (respectively, $\mathrm{X}<\mathrm{Y}$ ) means that $X-Y$ is positive definite (respectively, negative definite). $A^{T}$ is the transpose of the matrix $A$. $\operatorname{diag}\{\ldots\}$ represents a block-diagonal matrix. "*" denotes the symmetric term in a symmetric matrix. The symbol $\lambda_{\min }(X)$ means the minimum eigenvalue of a invertible matrix $X$. For a square matrix $X$, one has $\operatorname{He}(X)=X+X^{T}$.

\section{Problem Formulation and Preliminaries}

2.1. Stochastic Unmanned Surface Vehicles Model. As depicted in Figure $1, v, r, p, \delta, \varphi$, and $\phi$ represent the sway velocity, yaw velocity, roll velocity, rudder angle, heading angel, and roll angel, respectively, besides these variables, 


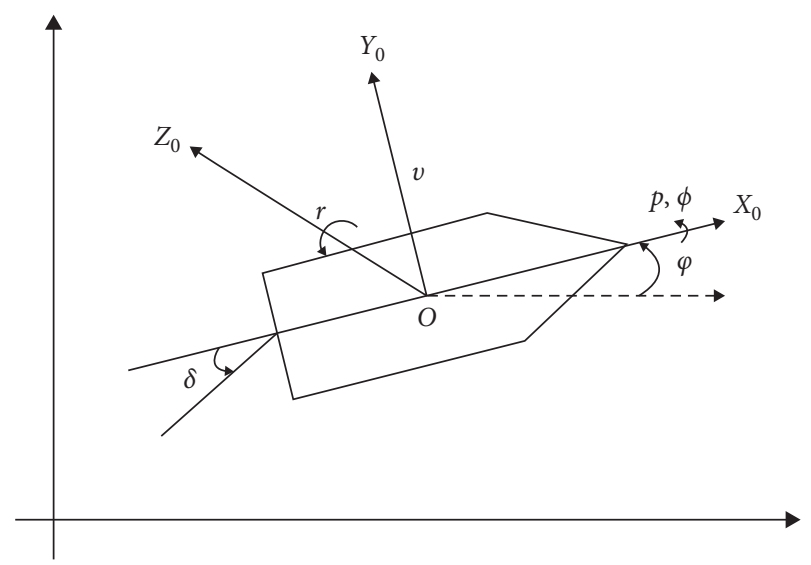

Figure 1: Motion coordinate system for SUSVs.

$X_{0}, Y_{0}$, and $Z_{0}$ denote the longitudinal axis, transverse axis, and normal axis, respectively.

$$
\left\{\begin{array}{l}
v(s)=\frac{K_{\theta(t)}^{\mathrm{d} v}}{1+T^{v} s} \delta(s), \\
\varphi(s)=\frac{1}{1+T^{r} s}\left[K_{\theta(t)}^{\mathrm{d} r} \delta(s)+K_{\theta(t)}^{v r} v(s)+\omega_{\varphi}(s)\right], \\
\phi(s)=\frac{\omega_{n}^{2}}{s^{2}+2 \zeta \omega_{n} s+\omega_{n}^{2}}\left[K_{\theta(t)}^{\mathrm{d} p} \delta(s)+K_{\theta(t)}^{v p} v(s)+\omega_{\phi}(s)\right],
\end{array}\right.
$$

Using the similar technique as in [5], the transfer functions for the stochastic unmanned surface vehicles (SUSVs) model is derived as follows:where $\omega_{\varphi}$ and $\omega_{\phi}$ indicate the effect of the wave on $\varphi$ and $\phi$, respectively; $\zeta$ is the damping ratio, and $\omega_{n}$ is the natural frequency under no damping; both $T^{v}$ and $T^{r}$ denote the time constants; and $K_{\theta(t)}^{v r}, K_{\theta(t)}^{v p}, K_{\theta(t)}^{\mathrm{d} v}, K_{\theta(t)}^{\mathrm{d} r}$, and $K_{\theta(t)}^{\mathrm{d} p}$ stand for the uncertain parameters with the Markov random process $\theta(t)$.

The parameter $\{\theta(t)\}$ is the stochastic Markov process with right continuous trajectories and takes values in a finite set $N=\{1,2, \ldots, N\}$, which is subject to the transition probability matrix $\mathbb{S}=\left\{\mathcal{O}_{i j}\right\}, \forall i, j \in N$, and the mode transition probability are defined as follows:

$$
\operatorname{Pr}\{\theta(t+\Delta t)=j \mid \theta(t)=i\}= \begin{cases}\mathcal{O}_{i j} \Delta_{t}+o\left(\Delta_{t}\right), & i \neq j, \\ 1+\mathcal{O}_{i j} \Delta_{t}+o\left(\Delta_{t}\right), & i=j,\end{cases}
$$

where $\quad \lim _{\Delta_{t} \longrightarrow 0}\left(o\left(\Delta_{t}\right) / \Delta_{t}\right)=0, \mathcal{O}_{i j} \geq 0(i \neq j), \mathcal{O}_{i j}=$ $-\sum_{j=1, j \neq i}^{N} \mathcal{O}_{i j}$, and $\mathscr{O}_{i j}$ denotes the probabilities from mode $i$ at time $t$ to mode $j$ at time $t+\Delta_{t}$.

Remark 1. Note that when $\mathbb{S}=I$, the proposed SUSVs model will be reduced to the traditional USVs model as introduced in $[5,44]$. In other words, the considered model of USV in $[5,44]$ is a special case of our research.
Denoting $\quad x(t)=\left[\begin{array}{lllll}v(t) & r(t) & \varphi(t) & p(t) & \phi(t)\end{array}\right]^{T}$, $\omega(t)=\left[\begin{array}{ll}\omega_{\varphi}(t) & \omega_{\phi}(t)\end{array}\right]^{T}$, and $A_{\theta(t)}, B_{\theta(t)}, W$ are given as follows:

$$
A_{\theta(t)}=\left[\begin{array}{ccccc}
-\frac{1}{T^{v}} & 0 & 0 & 0 & 0 \\
\frac{K_{\theta(t)}^{v r}}{T^{r}} & -\frac{1}{T^{r}} & 0 & 0 & 0 \\
0 & 1 & 0 & 0 & 0 \\
\omega_{n}^{2} K_{\theta(t)}^{v p} & 0 & 0 & -2 \zeta \omega_{n} & -\omega_{n}^{2} \\
0 & 0 & 0 & 1 & 0
\end{array}\right],
$$$$
B_{\theta(t)}=\left[\begin{array}{c}
\frac{K_{\theta(t)}^{\mathrm{d} v}}{T^{v}} \\
\frac{K_{\theta(t)}^{\mathrm{d} r}}{T^{r}} \\
0 \\
\omega_{n}^{2} K_{\theta(t)}^{\mathrm{d} p} \\
0
\end{array}\right],
$$

$$
W=\left[\begin{array}{cc}
0 & 0 \\
\frac{1}{T^{r}} & 0 \\
0 & 0 \\
0 & \omega_{n}^{2} \\
0 & 0
\end{array}\right],
$$

one can obtain the following state-space model:

$$
\dot{x}(t)=A_{\theta(t)} x(t)+B_{\theta(t)} \delta(t)+W \omega(t) .
$$

The corresponding structure of the proposed networked stochastic unmanned surface vehicles (NSUSVs) is shown in Figure 2. Note that the proposed NSUSVs suffer from actuator failures including bias fault and partial fault, external disturbance, asynchronization problem among original system, controller, and actuator as well as AETM with adaptive threshold.

2.2. The Mode-Dependent Adaptive Event-Triggered Mechanism. In this paper, the event detector is used to determine whether the present sampling data should be 


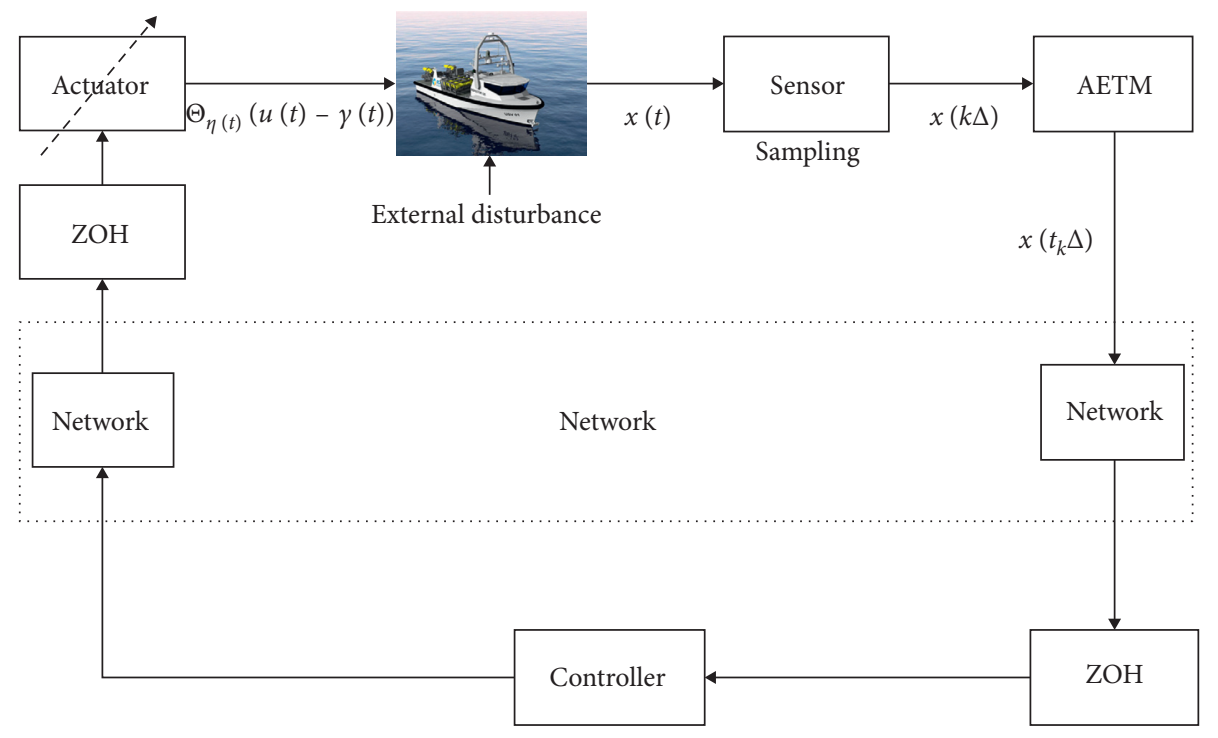

FIGURE 2: The structure of NSUSVs.

stored and sent out to the controller, and the event is triggered if the following inequality is satisfied:

$$
\begin{aligned}
& {\left[x\left(t_{k} \Delta+l \Delta\right)-x\left(t_{k} \Delta\right)\right]^{T} \Omega_{\theta(t)}\left[x\left(t_{k} \Delta+l T\right)-x\left(t_{k} \Delta\right)\right]} \\
& >\sigma(t) x^{T}\left(t_{k} \Delta+l \Delta\right) \Omega_{\theta(t)} x\left(t_{k} \Delta+l \Delta\right)
\end{aligned}
$$

where $\Delta$ is the sampling period of the sampler and the positive definite mode-dependent matrix $\Omega_{\theta(t)}$ is the weighting matrix to be designed later. $x(k \Delta)$ is the current sampling state at sampling instant $k \Delta\left(k \Delta \in R_{1} \triangleq\{0,1 \Delta, 2 \Delta, \ldots\}\right)$, and $x\left(t_{k} \Delta\right)$ is the latest transmitted state at the releasing instant $t_{k} \Delta\left(t_{k} \Delta \in R_{2} \triangleq\left\{t_{0} \Delta, t_{1} \Delta, t_{2} \Delta, \ldots\right\}\right)$, as well as we assume the first releasing instant $t_{0}=0$. On the other hand, $\sigma(t)$ is the event-triggered threshold variable, which meets the following adaptive law concerning event-triggered strategy:

$$
\dot{\sigma}(t) \triangleq \begin{cases}0, & \sigma(t) \longrightarrow 0^{+}, \\ \frac{1}{\sigma(t)}\left[\frac{1}{\sigma(t)}-\vartheta\right] e^{T}(t) \Omega_{\theta(t)} e(t), & \text { otherwise, }\end{cases}
$$

where $\quad 0<\sigma(t)<1,9>0 . e(t)=x\left(t_{k} \Delta+l \Delta\right)-x\left(t_{k} \Delta\right)$, $\forall l=1,2, \ldots, \ell$. The term $t_{k} \Delta+l \Delta$ denotes the sampling instants from the current transmitted sampling instant $t_{k} \Delta$ to the future transmitted sampling instant $t_{k+1} \Delta$. Note that the next releasing instant $t_{k+1} \Delta=t_{k} \Delta+\ell \Delta$ if condition (4) is satisfied.

We denote

$$
\Xi_{t_{k}}=\left[t_{k} \Delta+d_{t_{k}}, t_{k+1} \Delta+d_{t_{k+1}}\right]=\bigcup_{l=1}^{\ell} \Xi_{l, t_{k}},
$$

where $\Xi_{l, t_{k}}=\left[t_{k} \Delta+(l-1) \Delta+d_{t_{k}+(l-1)}, t_{k} \Delta+l \Delta+d_{t_{k}+l}\right)$.

Define the network induced delay as

$$
d(t)=t-t_{k} \Delta-l \Delta, \quad t \in \bigcup_{l=1}^{\ell} \Xi_{l, t_{k}} .
$$

Noting that the network-induced time-delay is represented as the maximum allowable upper communication delay bound $\bar{d}$ which is similar to [45], that is

$$
d_{m}=\min \left\{d_{t_{k}}\right\} \leq d(t) \leq 1+\bar{d}=d_{M}, \quad \forall t \in \bigcup_{l=1}^{\ell} \Xi_{l, t_{k}} .
$$

Finally, the AETM is deduced as follows:

$$
e^{T}(t) \Omega_{\theta(t)} e(t) \leq \sigma(t) x^{T}(t-d(t)) \Omega_{\theta(t)} x(t-d(t)) .
$$

Figure 3 gives an example to demonstrate the relationship between the sampling instant and the releasing instant, where the data are sampled discretely at instant $k \Delta\left(k \Delta \in R_{1} \triangleq\{0,1 \Delta, 2 \Delta, \ldots\}\right)$ and the data are chosen at instant $t_{k} \Delta\left(t_{k} \Delta \in R_{2} \triangleq\left\{t_{0} \Delta, t_{1} \Delta, t_{2} \Delta, \ldots\right\}\right)$ to release into the network. Based on the above analysis, we know that $R_{2} \subseteq R_{1}$. Besides, the control input of the proposed NSUSVs is reserved by $\mathrm{ZOH}$ during the time interval $\Xi_{1,0}=\left[0 \Delta+d_{0}, 3 \Delta+d_{3}\right), \Xi_{2,3}=\left[3 \Delta+d_{3}, 5 \Delta+d_{5}\right), \Xi_{3,5}=$ $\left[5 \Delta+d_{5}, t_{3} \Delta+4 \Delta+d_{t_{3}+4}\right), \ldots$.

Remark 2. Note that the mode-dependent AETM proposed in this paper relies on modes information of the system, which can efficiently reduce the loss of data in the network transmission, and it is not taken into account in most of the existing literature; see, for instance, [23, 24, 31, 45, 46].

Remark 3. Note that when $\dot{\sigma}(t)=0$, the supposed AETM can be degraded into the traditional ones, in which the event-triggered threshold is a preset constant, such as $[23,24,31,45]$ with the following form:

$$
e^{T}(t) \Omega_{\theta(t)} e(t) \leq \sigma_{0} x^{T}(t-d(t)) \Omega_{\theta(t)} x(t-d(t)),
$$

where $\sigma_{0} \in(0,1]$. And the above scheme is also applicable if $\sigma(t) \longrightarrow 0^{+}$. 


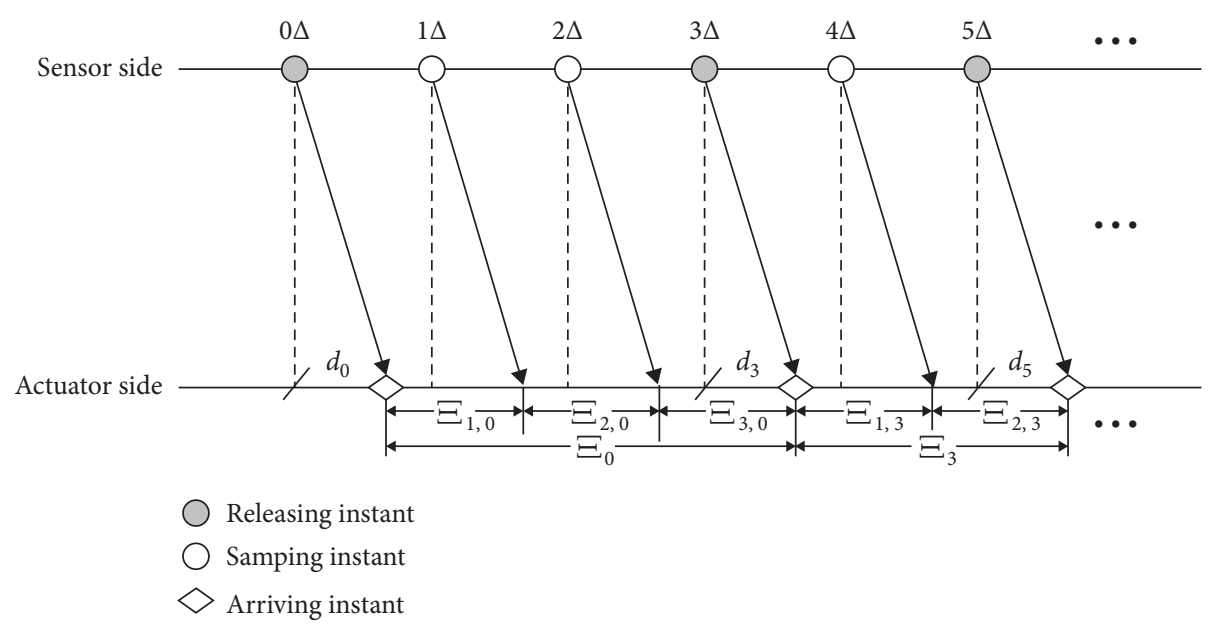

Figure 3: An example of timing diagram for AETM.

Remark 4. It is worth noting that the smallest interevent time can be regarded as the sampling period $\Delta$, so Zeno behavior will not exist in the related AETM proposed in this article.

2.3. Actuator Faults Analysis. The control input used by the NSUSVs with single rudder is described as follows:

$$
\delta(t)=\Theta_{\eta(t)}(u(t)-\gamma(t)),
$$

where $\Theta_{\eta(t)}$ represents the mode-dependent efficiency coefficient of actuator faults. $\Theta_{\eta(t)}^{\min }$ and $\Theta_{\eta(t)}^{\max }$ represent the unknown lower and upper bounds of $\Theta_{\eta(t)}$, that is, $0 \leq \Theta_{\eta(t)}^{\min } \leq \Theta_{\eta(t)} \leq \Theta_{\eta(t)}^{\max } \leq 1$, and $u(t)$ and $\gamma(t)$ are the applied control signal to be designed and the unknown time-varying bounded bias fault in the actuator, respectively. In addition, the jumps of actuator are under the control of Markov parameter $\eta(t)$, which are affected by the mode of system through conditional probability matrix $\mathbb{A}=\left\{\mathscr{P}_{i p}\right\}, \quad \forall i \in N$, and $p \in L=[1,2, \ldots, L]$. The conditional probability $\mathscr{P}_{i p}$ implies the possibility that the actuator runs in mode $p$ given the mode $i$ information of system, i.e.,

$$
\operatorname{Pr}\{\eta(t)=p \mid \theta(t)=i\}=\mathscr{P}_{i p}
$$

and for $\forall i \in N, p \in L$, there exist constraints $\mathscr{P}_{i p} \in[0,1]$ and $\sum_{p=1}^{L} \mathscr{P}_{i p}=1$.

For convenience, the subscript $p$ will be hereinafter employed to replace this Markov parameter $\eta(t)$ in the actuator, and the following multiple types of actuator faults model can be rewritten as

$$
\delta(t)=\Theta_{p}(u(t)-\gamma(t)) .
$$

From Table 1, one can see that when $\Theta_{p}=1$ and $\gamma(t)=0$, the actuator works normally. Both $\Theta_{p}=1$ and $\gamma(t) \neq 0$ mean the bias fault has appeared in the actuator. $0<\Theta_{p}<1$ and $\gamma(t)=0$ denote the partial fault has existed in the actuator. $0<\Theta_{p}<1$ and $\gamma(t) \neq 0$ represent the actuator that has occurred mixed failures including the partial fault and the bias fault. Regardless of whether $\gamma(t)$ is equal to 0 or not, the actuator outage fault has occurred in the actuator under $\Theta_{p}=0$.
TABLe 1: Fault models.

\begin{tabular}{lcc}
\hline & $\gamma(t)=0$ & $\gamma(t) \neq 0$ \\
\hline$\Theta_{p}=1$ & Normal & Bias \\
$\Theta_{p} \in(0,1)$ & Partial & Partial and bias \\
$\Theta_{p}=0$ & Outage & Outage \\
\hline
\end{tabular}

Using a similar method as [32], that is, by defining $\Theta_{p}=$ $\left(I+Z_{p}\right) \bar{\Theta}_{p}$ and $\left|Z_{p}\right| \leq H_{p} \leq I$, the conservatism that $\Theta_{p}$ is not known in advance is moderated, where

$$
\begin{aligned}
& \bar{\Theta}_{p}=\frac{\Theta_{p}^{\max }+\Theta_{p}^{\min }}{2}, \\
& H_{p}=\frac{\Theta_{p}^{\max }-\Theta_{p}^{\min }}{\Theta_{p}^{\max }+\Theta_{p}^{\min },} \\
& Z_{p}=\frac{\Theta_{p}-\bar{\Theta}_{p}}{\bar{\Theta}_{p}}, \quad \forall p \in L .
\end{aligned}
$$

The following assumptions are necessary to facilitate the subsequent research.

Assumption 1. For state-space model (4), in order to make the disturbance and input signal satisfy the match condition, there are constant matrix $C_{i}$ with appropriate dimensions such that

$$
W=B_{i} C_{i}
$$

Assumption 2. For the actuator partial fault $\Theta_{p}, \forall i \in N, p \in L$, one has

$$
\operatorname{rank}\left[B_{i} \Theta_{p}\right]=\operatorname{rank}\left[B_{i}\right] .
$$

Assumption 3. Define the augmented disturbance as follows:

$$
\psi(t)=-\Theta_{p} \gamma(t)+C_{i} \omega(t),
$$


where $\underline{\psi} \leq \psi(t) \leq \bar{\psi}$ and $\underline{\psi}$ as well as $\bar{\psi}$ are unknown constants.

One is defined as follows:

$$
\Psi=\|\underline{\psi}+\bar{\psi}\| .
$$

2.4. Asynchronous Adaptive Fault-Tolerant Controller. Let $\widehat{\Theta}_{p}(t), \widehat{\Psi}(t), \widehat{\bar{\psi}}(t), \widehat{\psi}(t)$ denote the online estimation of $\Theta_{p}, \Psi, \bar{\psi}, \underline{\psi}$, respectively.

One can define

$$
\partial(t)= \begin{cases}0, & x^{T}(t) \mathscr{P}_{i} B_{i} \widehat{\Theta}_{p}(t) \mu^{-1}(t)>0 \\ 1, & x^{T}(t) \mathscr{P}_{i} B_{i} \widehat{\Theta}_{p}(t) \mu^{-1}(t) \leq 0\end{cases}
$$

where $\mu(t)=\widehat{\Theta}_{p}(t)+\rho(t)$ and $\rho(t)$ is an exponential function and is defined as follows:

$$
\rho(t)=a e^{-b t}
$$

where both $a$ and $b$ are bounded positive bound constants to be designed. It can be figured out directly $\int_{0}^{\infty} \rho(\tau) \mathrm{d} \tau \leq \bar{\rho}<\infty$

Remark 5. It is worth noting that the introduction of $\rho(t)$ aims to use variable $\mu^{-1}(t)=\left(\widehat{\Theta}_{p}(t)+\rho(t)\right)^{-1}$ substitute for $\widehat{\Theta}_{p}^{-1}(t)$ in order to compensate the actuator partial fault $\Theta_{p}$. The reason for adopting this method is that we should consider the case of $\Theta_{p}=0$ and also analyze the outage fault of the actuator (for detail, see Table 1$)$, that is, $\widehat{\Theta}_{p}(t)=0$. Hence, $\widehat{\Theta}_{p}^{-1}(t)$ will be not defined well. So the introduction of parameter $\rho(t)$ is indispensable, which is not considered in $[29,34]$.

Next, we define the vector-valued function as follows:

$$
\omega(t)=\widehat{\bar{\psi}}(t)+\partial(t)(\underline{\hat{\psi}}(t)-\widehat{\bar{\psi}}(t)) .
$$

The asynchronous adaptive fault-tolerant controller under AETM is designed as follows:

$$
u(t)=\left(K_{1 \xi(t)}+K_{2 \xi(t)}\right) x\left(t_{k} \Delta\right)+K_{3 \xi(t)} \bowtie(t),
$$

where $K_{1 \xi(t)}, K_{2 \xi(t)}$, and $K_{3 \xi(t)}$ are the gains of the proposed mode-dependent fault-tolerant controller, which will be designed in the sequel. Markov parameter $\xi(t)$ exerts influence on the variations of the controller among different modes; on the other hand, they are affected by the mode of system through conditional probability matrix $\mathbb{C}=\left\{\mathcal{Q}_{i q}\right\}$ for $\forall i \in N, q \in M=[1,2, \ldots, M]$. The conditional probability $Q_{i q}$ implies the possibility that the controller runs in mode $q$ given the mode information $i$ of system, i.e.,

$$
\operatorname{Pr}\{\xi(t)=q \mid \theta(t)=i\}=Q_{i q},
$$

and it is extremely obvious that $Q_{i q} \in[0,1]$ and $\sum_{q=1}^{M} Q_{i q}=1$ for $\forall i \in N, q \in M$.

Remark 6. The implication of mode-dependent in this paper is that the actuator and controller depend indirectly on the NSUSVs mode $\theta(t)$ that is subject to transition probability (2) through conditional probabilities (13) and (24).

Remark 7. There exist problems such as packet loss, time delay, and quantization in NSUSVs, which will result in the incomplete information transfer. Therefore, the controller's modes information which is represented by $\xi(t)$ (subject to the conditional probability matrix $\mathbb{C}$ ) run asynchronously with the NSUSVs' modes information which is represented by $\theta(t)$ (subject to the transition probability matrix $\mathbb{S}$ ), but the latter directly imposes influence on the former. The asynchronization between the NSUSVs and controller is described by the hidden Markov model $[\theta(t), \xi(t), \mathbb{S}, \mathbb{C}$,$] . Similarly, the hidden Markov model$ $[\theta(t), \eta(t), \mathbb{S}, \mathbb{A}]$ is made up, which accounts for the asynchronization between the NSUSVs and actuator.

Remark 8. Note that similar to the description of HMM in [14], taking different values in the results will reduce to the following three cases:

(1) When $\mathbb{C}=I$, the controller modes are completely synchronous with the NSUSVs modes.

(2) Similar with the description in [22], the Markov parameter $\theta(t)$ of the system is grouped into several clusters, and in each cluster, the conditional probability $\mathbb{Q}_{i q}$ for $\xi(t)$ solely relies on which cluster $\theta(t)$ belongs to. One extreme case is that there is only one cluster and then the conditional probability matrix $\mathbb{C}$ has identical rows.

(3) When $\xi(t) \in\{1\}$ and $\mathbb{C}=[1 \ldots 1]^{T}$, the mode information $\theta(t)$ of system fail to work, in other word, the case is referred to as mode-independent.

For notational brevity again, the subscripts $i, j, q$ will be hereinafter employed to replace these Markov parameters $\theta(t), \theta\left(t+\Delta_{t}\right)$, and $\xi(t)$ in the system and controller. So, the dynamics of the closed-loop NSUSVs are obtained as follows:

$$
\begin{array}{r}
\dot{x}(t)=A_{i} x(t)+B_{i} \Theta_{p} K_{1 q} x(t-d(t))-B_{i} \Theta_{p} K_{1 q} e(t) \\
+B_{i} \Theta_{p} K_{2 q} x\left(t_{k} \Delta\right)+B_{i} \Theta_{p} K_{3 q} \omega(t)+B_{i} \psi(t) .
\end{array}
$$

Next, the adaptive laws for $\widehat{\Theta}_{p}(t)$ is designed as follows:

$$
\dot{\widehat{\Theta}}_{p}(t) \triangleq \operatorname{Proj}\left[\min \left\{\Theta_{p}\right\}, \max \left\{\Theta_{p}\right\}\right]\{\mathfrak{L}(t)\}= \begin{cases}0, & \text { if } \widehat{\Theta}_{p}=\min \left\{\Theta_{p}\right\}, \text { and } \mathfrak{L} \leq 0 \\ & \text { or } \widehat{\Theta}_{p}=\min \left\{\Theta_{p}\right\}, \text { and } \mathfrak{Q}>0, \\ \mathfrak{L}(t), & \text { otherwise, }\end{cases}
$$


where $\mathfrak{Q}(t)=-\tau_{\Theta} x^{T}(t) \mathscr{P}_{i} B_{i} \mu^{-1}(t) \omega(t)$.

Moreover, the adaptive laws for $\widehat{\Psi}(t), \widehat{\bar{\psi}}(t), \hat{\psi}(t)$ are designed as follows:

$$
\begin{aligned}
& \dot{\hat{\Psi}}(t)=\lambda_{\Psi}\|x(t)\|\left\|\mathscr{P}_{i} B_{i}\right\|\left\|\rho(t) \mu^{-1}(t)\right\|, \\
& \dot{\bar{\psi}}(t)=g_{\psi}(1-\partial(t)) x^{T}(t) \mathscr{P}_{i} B_{i} \widehat{\Theta}_{p}(t) \mu^{-1}(t), \\
& \dot{\hat{\psi}}(t)=g_{\psi} \partial(t) x^{T}(t) \mathscr{P}_{i} B_{i} \widehat{\Theta}_{p}(t) \mu^{-1}(t),
\end{aligned}
$$

and $\lambda_{\Psi}, \tau_{\Theta}, g_{\psi}$ are the adaptive law gains to be designed in the sequel.

Besides those parameters, $K_{2 q}$ and $K_{3 q}$ are designed as follows:

$$
\begin{aligned}
& K_{2 q}=-\frac{\left\|\rho(t) \mu^{-1}(t)\right\|^{2}\left\|\mathscr{P}_{i} B_{i}\right\|^{2} \widehat{\Psi}^{2}(t)}{\beta\left\|x\left(t_{k} \Delta\right)\right\|\left\|\rho(t) \mu^{-1}(t)\right\|\left\|\mathscr{P}_{i} B_{i}\right\| \widehat{\Psi}(t)+\rho(t)} B_{i}^{T} \mathscr{P}_{i}^{T}, \\
& K_{3 q}=-\mu^{-1}(t) .
\end{aligned}
$$

Our main purpose is to design an asynchronous adaptive fault-tolerant controller under AETM to ensure that uniformly ultimate boundedness of the solutions of the closedloop NSUSVs (25) can be achieved with adaptive law (6), (26), (27).

Before presenting our results, it is necessary to introduce the following lemmas.

Lemma 1 (Schur complement) (see [47]). Given a symmetric matrix as follows:

$$
S=\left[\begin{array}{ll}
S_{11} & S_{12} \\
S_{12}^{T} & S_{22}
\end{array}\right],
$$

and the following statements are equivalent:
(1) $S<0$
(2) $S_{11}<0, S_{22}-S_{12}^{T} S_{11}^{-1} S_{12}<0$
(3) $S_{22}<0, S_{11}-S_{12} S_{22}^{-1} S_{12}^{T}<0$

Lemma 2 (see [48]). Given matrices $\Phi, \Gamma$, and $\Lambda$ of appropriate dimensions with $\Phi$ being symmetrical, then $\Phi+$ $\Gamma F(t) \Lambda+\Lambda^{T} F^{T}(t) \Gamma^{T}<0$ for all $F(t)$ satisfying $F^{T}(t) F(t) \leq I$, if and only if there exists a scalar $\alpha>0$ such that $\Phi+\alpha \Gamma \Gamma^{T}+\alpha^{-1} \Lambda^{T} \Lambda<0$.

Lemma 3 (see [43]). For any partial fault parameter $\Theta_{p}$, the matrix $\mathscr{P}_{i} B_{i} \Theta_{p} B_{i}^{T} \mathscr{P}_{i}^{T}$ is invertible, and there exists a positive constant $\beta$ such that

$$
\mathscr{P}_{i} B_{i} \Theta_{p} B_{i}^{T} \mathscr{P}_{i}^{T} \geq \beta I
$$

Proof. Setting $r_{2}=\beta=\lambda_{\text {min }}\left(\mathscr{P}_{i} B_{i} \Theta_{p} B_{i}^{T} \mathscr{P}_{i}^{T}\right)$ in Lemma 2 in [43], the proof is obtained.

\section{Main Results}

This section will present a sufficient uniformly ultimate boundedness (for details, see definition 4.6 in [49]) condition for the closed-loop NSUSVs (25).

Theorem 1. For given positive scalars $d_{M}, d_{m}, \vartheta$, and matrix $K_{1 q}$, if there exist positive definite matrices $\Omega_{i}, G$, a positive definite symmetric matrix $\mathscr{P}_{i}, \forall i \in N, p \in L, q \in M$, such that the following conditions hold:

$$
\Pi=\left[\begin{array}{ccc}
\Pi_{11} & \Pi_{i p q} & -\Pi_{i p q} \\
* & \Omega_{i}-G & 0 \\
* & * & -\vartheta \Omega_{i}
\end{array}\right]<0,
$$

where

$$
\begin{aligned}
\Pi_{11} & =\mathscr{P}_{i} A_{i}+A_{i}^{T} \mathscr{P}_{i}+\left(d_{M}-d_{m}\right) G+\sum_{j=1}^{N} \mathcal{O}_{i j} \mathscr{P}_{j}, \\
\Pi_{i p q} & =\sum_{p=1}^{L} \mathscr{P}_{i p} \sum_{q=1}^{M} \mathscr{Q}_{i q} \mathscr{P}_{i} B_{i} \Theta_{p} K_{1 q} .
\end{aligned}
$$

The solutions of (25) are uniformly ultimate bounded with adaptive law (6), (26), (27).

Proof. Define the following error variables:

$$
\begin{aligned}
\widetilde{\Theta}_{p}(t) & =\widehat{\Theta}_{p}(t)-\Theta_{p}, \\
\tilde{\bar{\psi}}(t) & =\widehat{\bar{\psi}}(t)-\bar{\psi}, \\
\tilde{\psi}(t) & =\widehat{\psi}(t)-\underline{\psi}, \\
\widetilde{\Psi}(t) & =\widehat{\Psi}(t)-\Psi, \\
\Psi & =\|\underline{\psi}+\bar{\psi}\| .
\end{aligned}
$$

We introduce the following Lyapunov-Krasovskii functional:

$$
V(t)=\sum_{\hbar}^{6} V_{\hbar}(t),
$$

where

$$
\begin{aligned}
& V_{1}(t)=x^{T}(t) \mathscr{P}_{i} x(t), \\
& V_{2}(t)=\int_{t-d_{M}}^{t-d_{m}} \int_{t-1+z}^{t} x^{T}(y) G x(y) \mathrm{d} y \mathrm{~d} z, \\
& V_{3}(t)=\frac{1}{2} \sigma^{2}(t), \\
& V_{4}(t)=\frac{\widetilde{\Theta}_{p}^{2}(t)}{\tau_{\Theta}}, \\
& V_{5}(t)=\frac{\widetilde{\Psi}^{2}(t)}{\lambda_{\Psi}}, \\
& V_{6}(t)=\frac{1}{g_{\psi}}\left[\widetilde{\bar{\psi}}^{2}(t)+\underline{\Psi}^{2}(t)\right] .
\end{aligned}
$$


Based on the weak infinitesimal operator $\mathfrak{A}$, one obtains the differential of $V(t)$ along with the solution of (25).

One has firstly,

$$
\begin{aligned}
\mathfrak{A}\left\{V_{1}(t)\right\}= & x^{T}(t)\left[A_{i}^{T} \mathscr{P}_{i}+\mathscr{P}_{i} A_{i}\right] x(t)+x^{T}(t) \sum_{j=1}^{N} \mathcal{O}_{i j} \mathscr{P}_{j} x(t) \\
& +2 x^{T}(t) \mathscr{P}_{i} B_{i} \psi(t) \\
& +2 \sum_{p=1}^{L} \mathscr{P}_{i p} \sum_{q=1}^{M} Q_{i q} x^{T}(t) \mathscr{P}_{i} B_{i} \Theta_{p} K_{1 q} x(t-d(t)) \\
& -2 \sum_{p=1}^{L} \mathscr{P}_{i p} \sum_{q=1}^{M} \mathscr{Q}_{i q} x^{T}(t) \mathscr{P}_{i} B_{i} \Theta_{p} K_{1 q} e(t) \\
& +2 \sum_{p=1}^{L} \mathscr{P}_{i p} \sum_{q=1}^{M} Q_{i q} x^{T}(t) \mathscr{P}_{i} B_{i} \Theta_{p} K_{2 q} x\left(t_{k} \Delta\right) \\
& +2 \sum_{p=1}^{L} \mathscr{P}_{i p} \sum_{q=1}^{M} \mathscr{Q}_{i q} x^{T}(t) \mathscr{P}_{i} B_{i} \Theta_{p} K_{3 q} @(t),
\end{aligned}
$$

where

$$
\begin{aligned}
2 x^{T}(t) \mathscr{P}_{i} B_{i} \psi(t)= & 2 x^{T}(t) \mathscr{P}_{i} B_{i} \frac{\widehat{\Theta}_{p}(t)}{\widehat{\Theta}_{p}(t)+\rho(t)} \psi(t) \\
& +2 x^{T}(t) \mathscr{P}_{i} B_{i} \frac{\rho(t)}{\widehat{\Theta}_{p}(t)+\rho(t)} \psi(t) .
\end{aligned}
$$

Secondly,

$$
\begin{aligned}
\mathfrak{A} V_{2}(t)= & x^{T}(k)\left[\left(d_{M}-d_{m}\right) G\right] x(k) \\
& -\int_{-d_{M}+1}^{-d_{m}+1} x^{T}(t-1+z) G x(t-1+z) \mathrm{d} z .
\end{aligned}
$$

We can easily obtain that

$$
\begin{aligned}
\int_{-d_{M}+1}^{-d_{m}+1} x^{T}(t-1+z) G x(t-1+z) \mathrm{d} z & =\int_{t-d_{M}}^{t-d_{m}} x^{T}(z) G x(z) \mathrm{d} z \\
& \geq x^{T}(t-d(t)) G x(t-d(t)),
\end{aligned}
$$

so

$\mathfrak{A}\left\{V_{2}(t)\right\} \leq x^{T}(k)\left[\left(d_{M}-d_{m}\right) G\right] x(k)-x^{T}(t-d(t)) G x(t-d(t))$.

Thirdly, based on the adaptive law (6),

$$
\begin{aligned}
\mathfrak{A}\left\{V_{3}(t)\right\} & =\sigma(t) \dot{\sigma}(t) \\
& =\frac{1}{\sigma(t)} e^{T}(t) \Omega_{i} e(t)-\vartheta e^{T}(t) \Omega_{i} e(t) \\
& \leq x^{T}(t-d(t)) \Omega_{i} x(t-d(t))-\vartheta e^{T}(t) \Omega_{i} e(t) .
\end{aligned}
$$

Furthermore, according to (26) and (27), we can obtain $\mathfrak{A}\left\{V_{4}(t)\right\}=2 \frac{\widetilde{\Theta}_{p}(t) \dot{\widetilde{\Theta}}{ }_{p}(t)}{\tau_{\Theta}}=-2 \widetilde{\Theta}_{p}(t) x^{T}(t) \mathscr{P}_{i} B_{i} \mu^{-1} \varpi(t)$,

$$
\mathfrak{\mathcal { U }}\left\{V_{5}(t)\right\}=2 \frac{\widetilde{\Psi}(t) \dot{\widetilde{\Psi}}(t)}{\lambda_{\Psi}}=2 \widetilde{\Psi}(t)\|x(t)\|\left\|\mathscr{P}_{i} B_{i}\right\|\left\|\rho(t) \mu^{-1}(t)\right\| .
$$

Eventually, one derives

$$
\begin{aligned}
\mathfrak{A}\left\{V_{6}(t)\right\}= & 2\left[\frac{\tilde{\bar{\psi}}(t) \dot{\bar{\psi}}(t)}{g_{\psi}}+\frac{\tilde{\psi}(t) \underline{\tilde{\psi}}(t)}{g_{\psi}}\right] \\
= & 2\left[\frac{\tilde{\bar{\psi}}(t) g_{\psi}(1-\partial(t)) x^{T}(t) \mathscr{P}_{i} B_{i} \widehat{\Theta}_{p}(t) \mu^{-1}(t)}{g_{\psi}}+\frac{\tilde{\psi}(t) g_{\psi} \partial(t) x^{T}(t) \mathscr{P}_{i} B_{i} \widehat{\Theta}_{p}(t) \mu^{-1}(t)}{g_{\psi}}\right] \\
= & 2 \widehat{\Theta}_{p}(t) \mu^{-1}(t)[\tilde{\bar{\psi}}(t)+\partial(t)(\underline{\tilde{\psi}}(t)-\tilde{\bar{\psi}}(t))] x^{T}(t) \mathscr{P}_{i} B_{i} \\
= & 2 \widehat{\Theta}_{p}(t) \mu^{-1}(t)[\widehat{\bar{\psi}}(t)+\partial(t)(\underline{\hat{\psi}}(t)-\widehat{\bar{\psi}}(t))] x^{T}(t) \mathscr{P}_{i} B_{i} \\
& -2 \widehat{\Theta}_{p}(t) \mu^{-1}(t)[\bar{\psi}(t)+\partial(t)(\underline{\psi}(t)-\bar{\psi}(t))] x^{T}(t) \mathscr{P}_{i} B_{i} .
\end{aligned}
$$


On the one hand, according to Lemma 3 and reminding that $\Psi=\|\underline{\psi}+\bar{\psi}\|$, one obtains from (36), (37), and (43)

$$
\begin{aligned}
& 2 x^{T}(t) \mathscr{P}_{i} B_{i} \frac{\rho(t)}{\widehat{\Theta}_{p}(t)+\rho(t)} \psi(t)+2 \sum_{p=1}^{L} \mathscr{P}_{i p} \sum_{q=1}^{M} Q_{i q} x^{T}(t) \mathscr{P}_{i} B_{i} \Theta_{p} K_{2 q} x\left(t_{k} \Delta\right)+\mathfrak{A}\left\{V_{5}(t)\right\} \\
& =\frac{2 x^{T}(t) \mathscr{P}_{i} B_{i} \rho(t) \mu^{-1}(t) \psi(t)\left[\beta\left\|x\left(t_{k} \Delta\right)\right\|\left\|\rho(t) \mu^{-1}(t)\right\|\left\|\mathscr{P}_{i} B_{i}\right\| \widehat{\Psi}(t)+\rho(t)\right]}{\beta\left\|x\left(t_{k} \Delta\right)\right\|\left\|\rho(t) \mu^{-1}(t)\right\|\left\|\mathscr{P}_{i} B_{i}\right\| \widehat{\Psi}(t)+\rho(t)} \\
& -2 \sum_{p=1}^{L} \mathscr{P}_{i p} x^{T}(t) \mathscr{P}_{i} B_{i} \Theta_{p} \frac{\left\|\rho(t) \mu^{-1}(t)\right\|^{2}\left\|\mathscr{P}_{i} B_{i}\right\|^{2} \widehat{\Psi}^{2}(t)}{\beta\left\|x\left(t_{k} \Delta\right)\right\|\left\|\rho(t) \mu^{-1}(t)\right\|\left\|\mathscr{P}_{i} B_{i}\right\| \widehat{\Psi}(t)+\rho(t)} B_{i}^{T} \mathscr{P}_{i}^{T} x\left(t_{k} \Delta\right) \\
& +\frac{2 \widetilde{\Psi}(t)\|x(t)\|\left\|\mathscr{P}_{i} B_{i}\right\|\left\|\rho(t) \mu^{-1}(t)\right\|\left[\beta\left\|x\left(t_{k} \Delta\right)\right\|\left\|\rho(t) \mu^{-1}(t)\right\|\left\|\mathscr{P}_{i} B_{i}\right\| \widehat{\Psi}(t)+\rho(t)\right]}{\beta\left\|x\left(t_{k} \Delta\right)\right\|\left\|\rho(t) \mu^{-1}(t)\right\|\left\|\mathscr{P}_{i} B_{i}\right\| \widehat{\Psi}(t)+\rho(t)} \\
& \leq \frac{2 \widehat{\Psi}(t)\|x(t)\|\left\|\mathscr{P}_{i} B_{i}\right\|\left\|\rho(t) \mu^{-1}(t)\right\| \rho(t)}{\beta\left\|x\left(t_{k} \Delta\right)\right\|\left\|\rho(t) \mu^{-1}(t)\right\|\left\|\mathscr{P}_{i} B_{i}\right\| \widehat{\Psi}(t)+\rho(t)} \\
& =\frac{2 \widehat{\Psi}(t)\left\|x\left(t_{k} \Delta\right)\right\|\left\|\mathscr{P}_{i} B_{i}\right\|\left\|\rho(t) \mu^{-1}(t)\right\| \rho(t)}{\beta\left\|x\left(t_{k} \Delta\right)\right\|\left\|\rho(t) \mu^{-1}(t)\right\|\left\|\mathscr{P}_{i} B_{i}\right\| \widehat{\Psi}(t)+\rho(t)} \\
& \leq 2 \beta^{-1} \rho(t) \text {. }
\end{aligned}
$$

On the other hand, from (36), (37), and (44), one gets

$$
\begin{aligned}
& 2 x^{T}(t) \mathscr{P}_{i} B_{i} \frac{\widehat{\Theta}_{p}(t)}{\widehat{\Theta}_{p}(t)+\rho(t)} \psi(t)+2 \sum_{p=1}^{L} \mathscr{P}_{i p} \sum_{q=1}^{M} \mathscr{Q}_{i q} x^{T}(t) \mathscr{P}_{i} B_{i} \Theta_{p} K_{3 p} \omega(t)+\mathfrak{A}\left\{V_{6}(t)\right\} \\
& \leq 2 x^{T}(t) \mathscr{P}_{i} B_{i} \widehat{\Theta}_{p}(t) \mu^{-1}(t) \psi(t) \\
& -2 \sum_{p=1}^{L} \mathscr{P}_{i p} \sum_{q=1}^{M} Q_{i q} x^{T}(t) \mathscr{P}_{i} B_{i} \widehat{\Theta}_{p}(t) \mu^{-1}(t)[\widehat{\bar{\psi}}(t)+\partial(t)(\underline{\hat{\psi}}(t)-\widehat{\bar{\psi}}(t))] \\
& +2 \sum_{p=1}^{L} \mathscr{P}_{i p} \sum_{q=1}^{M} Q_{i q} x^{T}(t) \mathscr{P}_{i} B_{i} \widetilde{\Theta}_{p}(t) \mu^{-1}(t)[\hat{\bar{\psi}}(t)+\partial(t)(\underline{\hat{\psi}}(t)-\widehat{\bar{\psi}}(t))] \\
& +2 \widehat{\Theta}_{p}(t) \mu^{-1}(t)[\widehat{\bar{\psi}}(t)+\partial(t)(\underline{\hat{\psi}}(t)-\widehat{\bar{\psi}}(t))] x^{T}(t) \mathscr{P}_{i} B_{i} \\
& -2 \widehat{\Theta}_{p}(t) \mu^{-1}(t)[\bar{\psi}(t)+\partial(t)(\underline{\psi}(t)-\bar{\psi}(t))] x^{T}(t) \mathscr{P}_{i} B_{i} \\
& \leq 2 x^{T}(t) \mathscr{P}_{i} B_{i} \widehat{\Theta}_{p}(t) \mu^{-1}(t)[\bar{\psi}(t)+\partial(t)(\underline{\psi}(t)-\bar{\psi}(t))] \\
& +2 \sum_{p=1}^{L} \mathscr{P}_{i p} x^{T}(t) \mathscr{P}_{i} B_{i} \widetilde{\Theta}_{p}(t) \mu^{-1}(t)[\hat{\bar{\psi}}(t)+\partial(t)(\underline{\hat{\psi}}(t)-\widehat{\bar{\psi}}(t))] \\
& -2 \sum_{p=1}^{L} \mathscr{P}_{i p} \widehat{\Theta}_{p}(t) \mu^{-1}(t)[\bar{\psi}(t)+\partial(t)(\underline{\psi}(t)-\bar{\psi}(t))] x^{T}(t) \mathscr{P}_{i} B_{i} \\
& =2 \sum_{p=1}^{L} \mathscr{P}_{i p} x^{T}(t) \mathscr{P}_{i} B_{i} \widetilde{\Theta}_{p}(t) \mu^{-1}(t)[\widehat{\bar{\psi}}(t)+\partial(t)(\underline{\hat{\psi}}(t)-\widehat{\bar{\psi}}(t))] .
\end{aligned}
$$


We $\chi(t)=\left[\begin{array}{lll}x^{T}(t) & x^{T}(t-d(t)) & e^{T}(t)\end{array}\right]^{T}$ to facilitate the following analysis. By combining (26) and (33)-(46), we endeavor to derive the following inequality:

$$
\begin{aligned}
\mathfrak{A}\{V(t)\}= & \mathfrak{A}\left\{\sum_{\hbar}^{6} V_{\hbar}(t)\right\} \\
\leq & \chi^{T}(t) \Pi \chi(t)-2 \widetilde{\Theta}_{p}(t) x^{T}(t) \mathscr{P}_{i} B_{i} \mu^{-1} \omega(t)+2 \beta^{-1} \rho(t) \\
& +2 \sum_{p=1}^{M} \psi_{i p} x^{T}(t) \mathscr{P}_{i} B_{i} \widetilde{\Theta}_{p}(t) \mu^{-1}(t) \\
& \cdot[\widehat{\bar{\psi}}(t)+\partial(t)(\underline{\psi}(t)-\widehat{\bar{\psi}}(t))] \\
\leq & \chi^{T}(t) \Pi \chi(t)+2 \beta^{-1} \rho(t) .
\end{aligned}
$$

Therefore, $\mathfrak{A}\{V(t)\}<0$ as long as it meets $\|\chi(t)\|>\sqrt{\left(2 \beta^{-1} \rho(t) /-\lambda_{\min }(\Pi)\right)}=\wp$. Hence, $\mathfrak{A}\{V(t)\}$ is negative definite outside the compact set $\Sigma_{\chi} \triangleq\{\chi \mid\|\chi(t)\| \geq \wp\}$, which represents that whatever $\|\chi(t)\|$ leaves the set $\Sigma_{\chi}, V(t)$ will decrease and $\|\chi(t)\|$ will still be bounded. Thus, one can conclude on the uniformly ultimate boundedness of the solutions of (25).

Remark 9. In Theorem 1, a sufficient condition is derived by introducing Lyapunov-Krasovskii functional (34) to guarantee the uniformly ultimate boundedness of the solutions of NSUSVs (25) with the multiple types of actuator faults and external disturbance. Moreover, the designed controller is based on the releasing state of adaptive event-triggered mechanism (5) and the online estimation of proposed augmented disturbance (18).

Next, we will design the proposed fault-tolerant controller gain $K_{1 q}$ for (25). The theorem is provided as follows.

Theorem 2. For given positive scalars $d_{M}, d_{m}, \vartheta, \varepsilon_{1}, \varepsilon_{2}, \varepsilon_{3}$, and a matrix $Y_{q}$, if there exist positive definite matrices $\bar{G}, \bar{\Omega}_{i}, \overline{\mathscr{P}}_{j}, \mathscr{L}$ with appropriate dimensions, such that the following LMI condition holds:

$$
\Phi_{i p q}=\left[\begin{array}{cc}
\Phi_{11} & \Phi_{12} \\
* & -\Phi_{22}
\end{array}\right]<0,
$$

where

$$
\begin{aligned}
\Phi_{11} & =\left[\begin{array}{ccc}
\bar{\Phi}_{11}+\mathscr{E}_{1} & \Phi_{11}^{i p q} & -\Phi_{11}^{i p q} \\
* & \bar{\Omega}_{i}-\bar{G}+\mathscr{E}_{2} & 0 \\
* & * & -\vartheta \bar{\Omega}_{i}+\mathscr{E}_{3}
\end{array}\right], \\
\bar{\Phi}_{11} & =A_{i} \mathscr{L}^{2} \mathscr{L}_{i}^{T}+\left(d_{M}-d_{m}\right) \bar{G}+\sum_{j=1}^{N} \mathcal{O}_{i j} \overline{\mathscr{P}}_{j}, \\
\Phi_{11}^{i p q} & =\sum_{p=1}^{L} \mathscr{P}_{i p} \sum_{q=1}^{M} Q_{i q} B_{i} \bar{\Theta}_{p} Y_{q}, \\
\mathscr{E}_{1} & =\varepsilon_{1} B_{i} H_{p} H_{p}^{T} B_{i}^{T}, \\
\mathscr{E}_{2} & =\varepsilon_{2} B_{i} H_{p} H_{p}^{T} B_{i}^{T}, \\
\mathscr{E}_{3} & =\varepsilon_{3} B_{i} H_{p} H_{p}^{T} B_{i}^{T}, \\
\Phi_{12} & =\Lambda^{T}=\left[\begin{array}{lll}
0 & \Phi_{12}^{i p q} & -\Phi_{12}^{i p q} \\
0 & 0 & 0 \\
0 & 0 & 0
\end{array}\right], \\
\Phi_{12}^{i p q} & =\sum_{q=1}^{M} \mathscr{Q}_{i q} \sum_{p=1}^{L} \mathscr{P}_{i p} Y_{q}^{T} \bar{\Theta}_{p}^{T}, \\
& \operatorname{diag}\left\{\varepsilon_{1}, \varepsilon_{2}, \varepsilon_{3}\right\} .
\end{aligned}
$$

Then, $K_{1 q}$ can be parameterized as follows:

$$
K_{1 q}=Y_{q} \mathscr{L}^{-1} \text {. }
$$

Proof. To begin with, we denote

$$
\begin{aligned}
L & =\mathscr{P}_{i}^{-1}, \\
Y_{q} & =\bar{K}_{1 q} \mathscr{L}, \\
\bar{G} & =\mathscr{L} G \mathscr{L}, \\
\bar{\Omega}_{i} & =\mathscr{L} \Omega_{i} \mathscr{L}, \\
\overline{\mathscr{P}}_{j} & =\mathscr{L} \mathscr{P}_{j} \mathscr{L},
\end{aligned}
$$

we denote $\mathbb{L}=\operatorname{diag}\{\mathscr{L}, \mathscr{L}, \mathscr{L}\}$, pre- and postmultiply (31) by $\mathbb{L}$, respectively, and then we get

$$
\bar{\Phi}_{i p q}=\left[\begin{array}{ccc}
\bar{\Phi}_{11} & \bar{\Phi}_{12}^{i p q} & -\bar{\Phi}_{12}^{i p q} \\
* & \bar{\Omega}_{i}-\bar{G} & 0 \\
* & * & -\vartheta \bar{\Omega}_{i}
\end{array}\right]<0,
$$

where $\bar{\Phi}_{12}^{i p q}=\sum_{p=1}^{L} \mathscr{P}_{i p} \sum_{q=1}^{M} Q_{i q} B_{i} \Theta_{p} Y_{q}$.

Because $\Theta_{p}=\left(I+Z_{p}\right) \bar{\Theta}_{p},\left|Z_{p}\right| \leq H_{p} \leq I$, so the following inequality is true:

$$
\bar{\Phi}_{i p q}=\widetilde{\Phi}_{i p q}+\Gamma F(t) \Lambda+\Lambda^{T} F(t) \Gamma^{T}, \quad \Gamma \Gamma^{T} \leq \overline{\Gamma \Gamma}^{T} .
$$

Thus, 


$$
\bar{\Phi}_{i p q} \leq \widetilde{\Phi}_{i p q}+\operatorname{He}(\bar{\Gamma} F(t) \Lambda)
$$

where

$$
\begin{aligned}
\widetilde{\bar{\Phi}}_{i p q} & =\left[\begin{array}{ccc}
\bar{\Phi}_{11} & \Phi_{11}^{i p q} & -\Phi_{11}^{i p q} \\
* & \bar{\Omega}_{i}-\bar{G} & 0 \\
* & * & -\vartheta \bar{\Omega}_{i}
\end{array}\right], \\
\Lambda^{T} & =\left[\begin{array}{ccc}
0 & \Phi_{12}^{i p q} & -\Phi_{12}^{i p q} \\
0 & 0 & 0 \\
0 & 0 & 0
\end{array}\right], \\
\Gamma^{T} & =\operatorname{diag}\left\{Z_{p}^{T} B_{i}^{T}, Z_{p}^{T} B_{i}^{T}, Z_{p}^{T} B_{i}^{T}\right\} \\
\bar{\Gamma}^{T} & =\operatorname{diag}\left\{H_{p}^{T} B_{i}^{T}, H_{p}^{T} B_{i}^{T}, H_{p}^{T} B_{i}^{T}\right\} \\
F(t) & =I .
\end{aligned}
$$

In accordance with Lemma 2 , for $F^{T}(t) F(t) \leq I$ if there exist positive scalar matrices $\Phi_{22}=\operatorname{diag}\left\{\varepsilon_{1}, \varepsilon_{2}, \varepsilon_{3}\right\}$ such that

$$
\widetilde{\Phi}_{i p q}+\Phi_{22} \overline{\Gamma \Gamma}^{T}+\Phi_{22}^{-1} \Lambda^{T} \Lambda<0
$$

meets.

Therefore, inequality (54) can be obtained further. Eventually, based on Lemma 1, inequality (48) can be derived from (51)-(56). This completes the proof.

Remark 10. Note that Theorem 2 provides a sufficient condition for the mode-dependent adaptive event-triggered matrix $\Omega_{\theta(t)}$ in the triggering condition (5) and the asynchronous adaptive fault-tolerant controller gain matrix $K_{1 \xi(t)}$ in equation (23). If the linear matrix inequalities (LMI) (48) is feasible, both $\Omega_{\theta(t)}$ and $K_{1 \xi(t)}$ can be easily figured out.

\section{Numerical Example}

In this section, the validity and availability of the proposed methods will be elaborated by a numerical example, where system modes $\theta(t) \in\{1,2\}$, actuator modes $\eta(t) \in\{1,2\}$, and controller modes $\xi(t) \in\{1,2\}$ are considered, and the relevant parameters are available from [44]:

$$
\begin{aligned}
U & =5.6 \frac{\mathrm{m}}{\mathrm{s}}, \\
T^{v} & =\frac{1.8}{U}, \\
T^{r} & =\frac{2}{U}, \\
\omega_{n} & =1.86\left(\frac{\mathrm{rad}}{\mathrm{s}}\right), \\
\zeta & =0.62+0.46 U, \\
K_{1}^{v r} & =-0.52,
\end{aligned}
$$

$$
\begin{aligned}
& K_{1}^{v p}=0.21 U, \\
& K_{1}^{\mathrm{d} v}=0.01 U, \\
& K_{1}^{\mathrm{d} r}=-0.003 U, \\
& K_{1}^{\mathrm{d} p}=-0.002 U^{2}, \\
& K_{2}^{v r}=-0.58, \\
& K_{2}^{v p}=0.16 U, \\
& K_{2}^{\mathrm{d} v}=0.016 U, \\
& K_{2}^{\mathrm{d} r}=-0.0036 U, \\
& K_{2}^{\mathrm{d} p}=-0.0022 U^{2},
\end{aligned}
$$

where $U$ is the forward speed of the USVs. Then, one educes

$$
A_{1}=\left[\begin{array}{ccccc}
-3.111 & 0 & 0 & 0 & 0 \\
-1.456 & -2.8 & 0 & 0 & 0 \\
0 & 1 & 0 & 0 & 0 \\
4.068 & 0 & 0 & -11.889 & -3.460 \\
0 & 0 & 0 & 1 & 0
\end{array}\right] \text {, }
$$$$
B_{1}=\left[\begin{array}{c}
0.174 \\
-0.047 \\
0 \\
-0.217 \\
0
\end{array}\right] \text {, }
$$

$$
A_{2}=\left[\begin{array}{ccccc}
-3.111 & 0 & 0 & 0 & 0 \\
-1.624 & -2.8 & 0 & 0 & 0 \\
0 & 1 & 0 & 0 & 0 \\
3.100 & 0 & 0 & -11.889 & -3.460 \\
0 & 0 & 0 & 1 & 0
\end{array}\right] \text {, }
$$

$$
W=\left[\begin{array}{cc}
0 & 0 \\
2.8 & 0 \\
0 & 0 \\
0 & 3.460 \\
0 & 0
\end{array}\right] \text {. }
$$


The transition probability matrix $\mathbb{S}$, along with conditional probability matrices $\mathbb{A}$ and $\mathbb{C}$, is selected as follows:

$$
\begin{aligned}
& \mathbb{S}=\left[\begin{array}{cc}
-0.75 & 0.75 \\
0.45 & -0.45
\end{array}\right], \\
& \mathbb{A}=\left[\begin{array}{cc}
0.65 & 0.35 \\
0.5 & 0.5
\end{array}\right], \\
& \mathbb{C}=\left[\begin{array}{cc}
0.55 & 0.45 \\
0.6 & 0.4
\end{array}\right] .
\end{aligned}
$$

Select the unknown partial failures as $0.3 \leq \Theta_{1} \leq 0.9,0.2 \leq \Theta_{2} \leq 0.8$, and the bias faults $\gamma(t)$ are as follows:

$$
\gamma(t)= \begin{cases}0, & 0 \leq t<5 \\ 0.1 \sin (0.35(t-5)), & t \geq 5\end{cases}
$$

In addition to those parameters, the network induced timedelay is chosen randomly from value 0.01 or 0.21 , that is, $d_{M}-d_{m}=0.2$ and $\vartheta=0.2$.

By solving the LMI in Theorem 2, we can obtain the adaptive fault-tolerant controller gain matrices

$$
\begin{aligned}
& K_{11}=\left[\begin{array}{lllll}
0.2234 & -0.2022 & 0.4622 & -0.3122 & -0.0773
\end{array}\right], \\
& K_{12}=\left[\begin{array}{lllll}
0.2426 & -0.2195 & 0.5018 & -0.3389 & -0.0839
\end{array}\right],
\end{aligned}
$$

and the mode-dependent event-triggered matrices

$$
\begin{aligned}
\Omega_{1} & =\left[\begin{array}{ccccc}
7.9307 & -0.0030 & 0 & -0.0128 & 0 \\
-0.0030 & 7.9160 & 0 & 0.0025 & 0 \\
0 & 0 & 7.9154 & 0 & 0 \\
-0.0128 & 0.0025 & 0 & 7.9259 & 0 \\
0 & 0 & 0 & 0 & 7.9154
\end{array}\right], \\
\Omega_{2} & =\left[\begin{array}{ccccc}
7.9195 & -0.0013 & 0 & -0.0059 & 0 \\
-0.0013 & 7.9158 & 0 & 0.0017 & 0 \\
0 & 0 & 7.9154 & 0 & 0 \\
-0.0059 & 0.0017 & 0 & 7.9235 & 0 \\
0 & 0 & 0 & 0 & 7.9154
\end{array}\right] .
\end{aligned}
$$

To perform some simulations about performances of NSUSVs, we suppose the initial condition $x(0)=\left[\begin{array}{lllll}0.1 & -0.5 & 0.4 & -0.3 & 0.6\end{array}\right]^{T}$ and the external interference input imposed on the system is $\omega(t)=\left[\begin{array}{lll}\omega_{\varphi}(t) & \omega_{\phi}(t)\end{array}\right]^{\mathrm{T}}=\left[\begin{array}{lll}0.2 \sin (3 t) & 0.4 \cos (4 t)\end{array}\right]^{T}$. Besides, the adaptive gains in (26) and (27) are set to be $\tau_{\Theta}=$ $0.8, g_{\psi}=0.5$, and $\lambda_{\Psi}=0.3$, respectively. The parameters $a$ and $b$ are designated as $a=1$ and $b=0.01$, so, we achieve $\rho(t)=e^{-0.01 t}$.

Based on the aforementioned parameters, we can obtain the simulation results demonstrated in Figures 4-11. The possible modes evolution of the NSUSVs, controller, and actuator is depicted in Figure 4, which obviously proves that controller and actuator run asynchronously with the
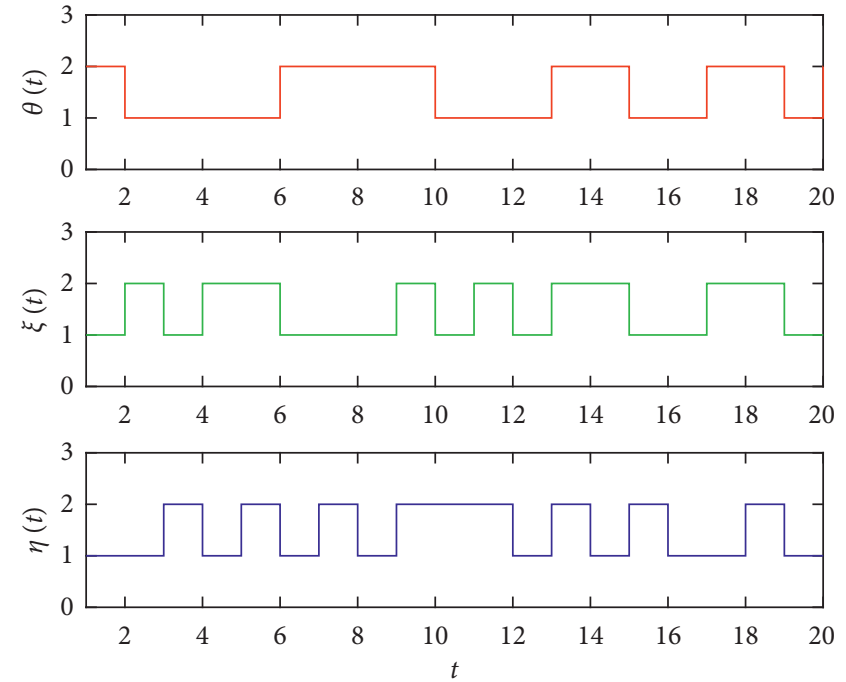

FIGURE 4: Modes evolution of the NSUSVs, controller, and actuator.

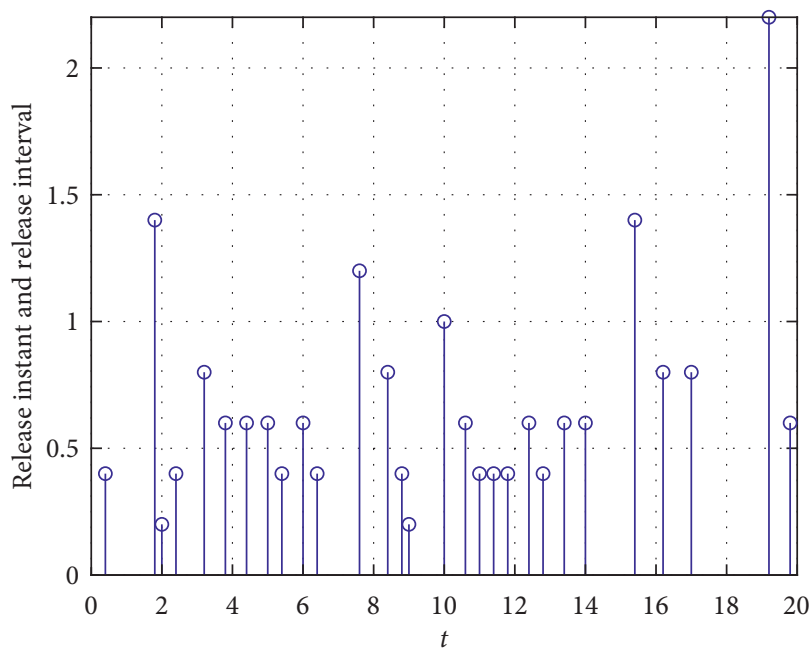

FIGURE 5: Release instants and release intervals.

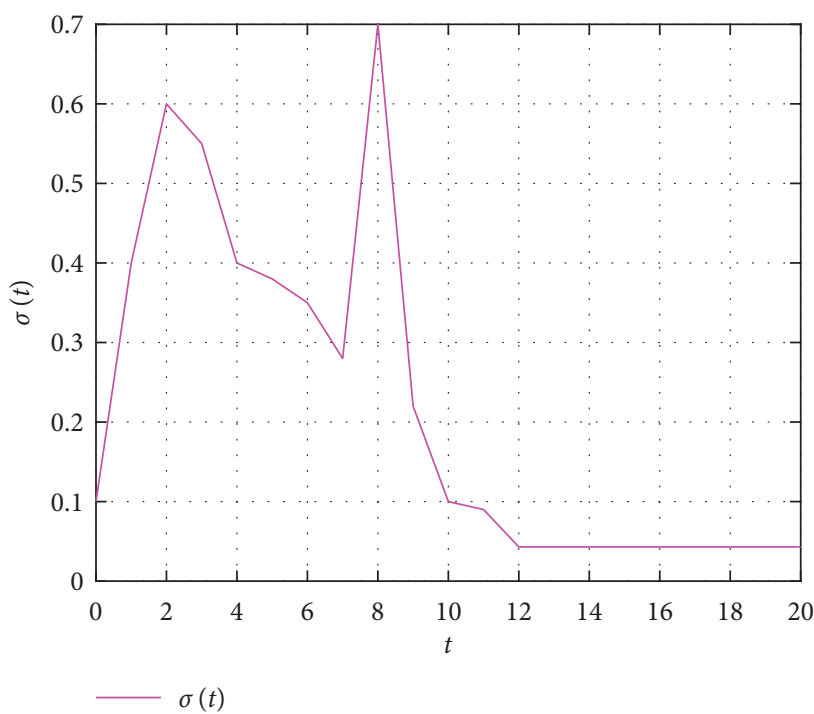

Figure 6: The threshold $\sigma(t)$ of AETM. 

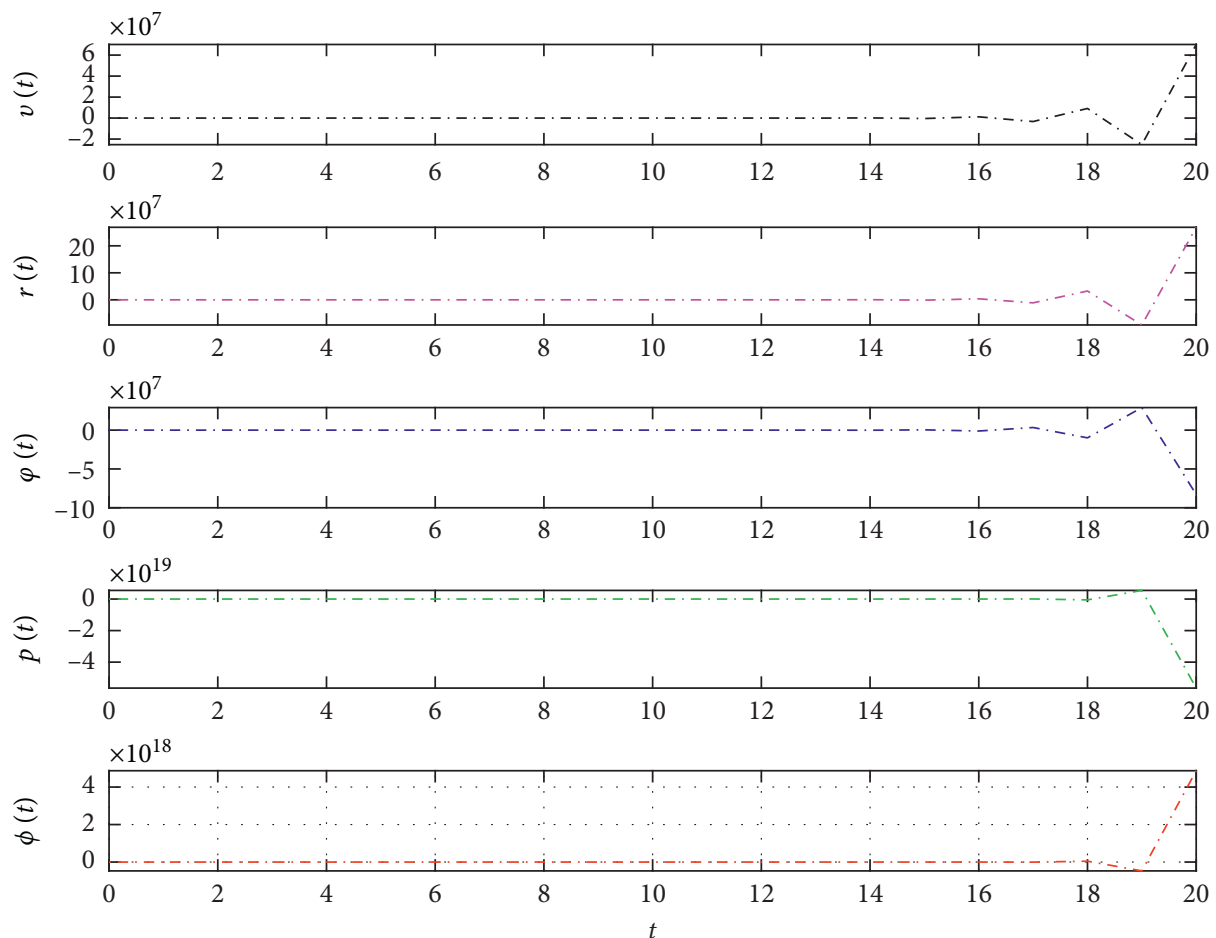

Figure 7: The state response of open-loop NSUSVs (25).
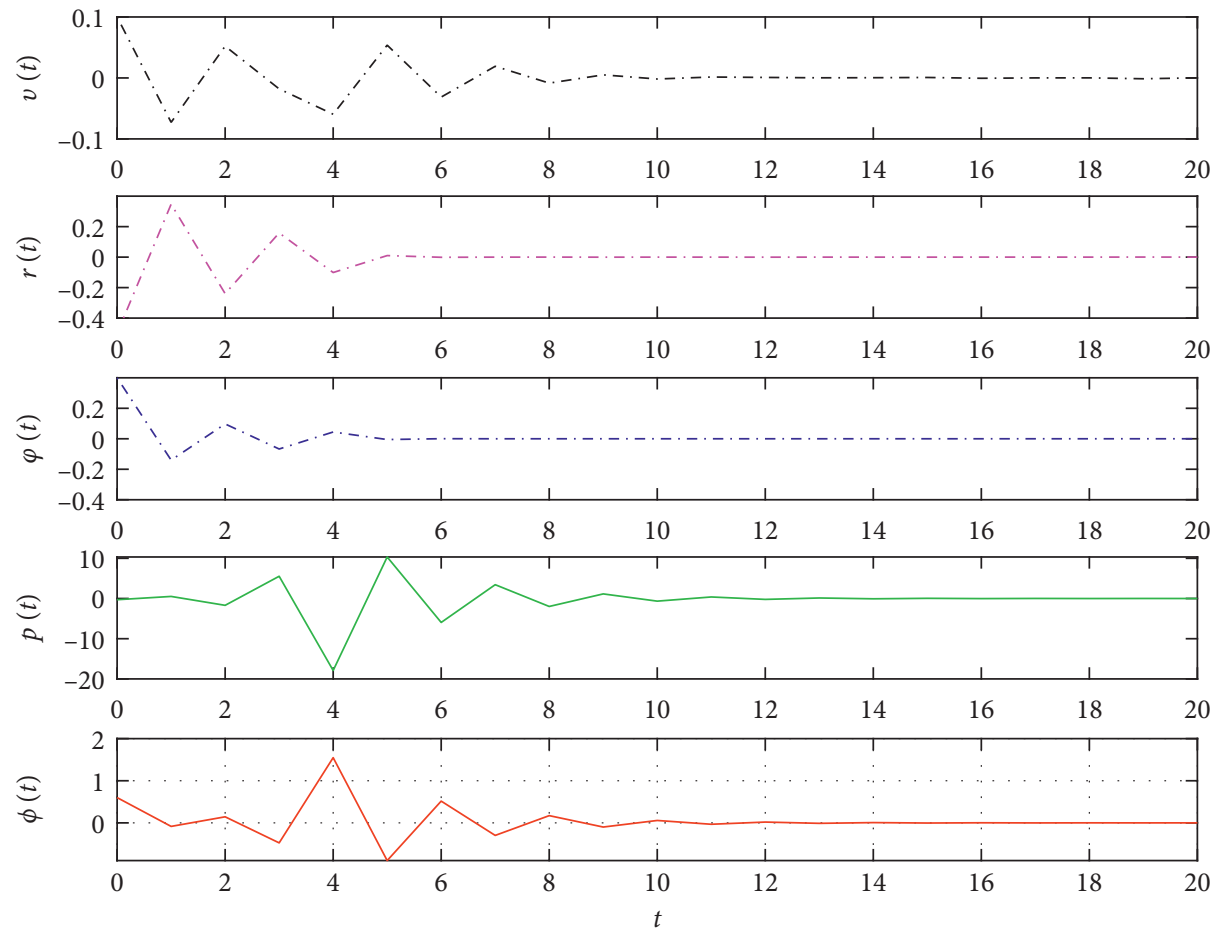

Figure 8: The state response of closed-loop of NSUSVs (25) by using our method.

NSUSVs since their variations among different modes are governed by three different parameters $\xi(t), \eta(t)$, and $\theta(t)$. The release instants and release intervals of the AETM (5) are displayed in Figure 5, which illustrates the availability and validity of the proposed AETM. Furthermore, the threshold $\sigma(t)$ of the AETM is described in Figure 6, and as can be seen from Figure 6 that $\sigma(t)$ eventually converges to 0.043 , which corresponds to the case $\sigma(t) \longrightarrow 0^{+}$. 

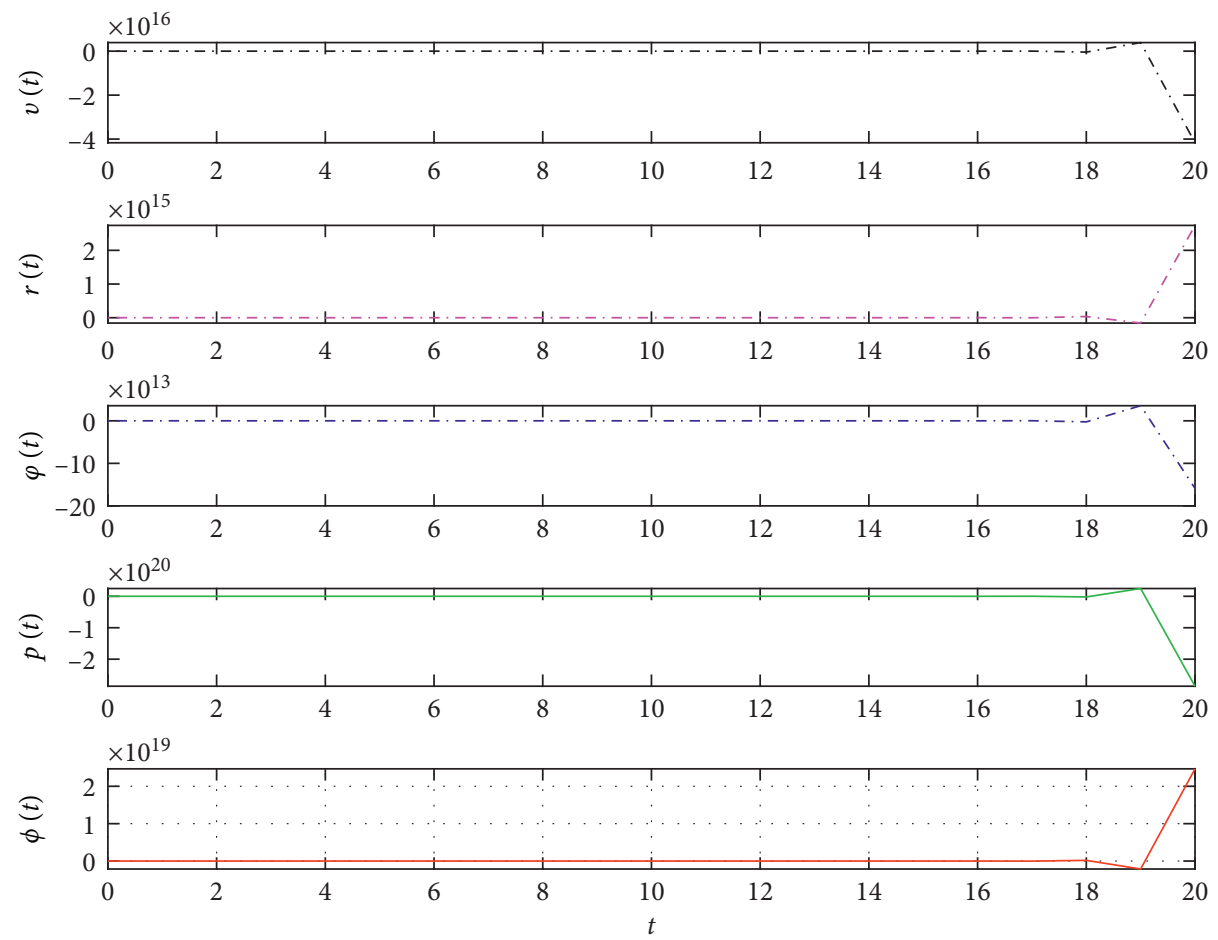

FIgURE 9: The state response of closed-loop of USVs by using the proposed method in [44].

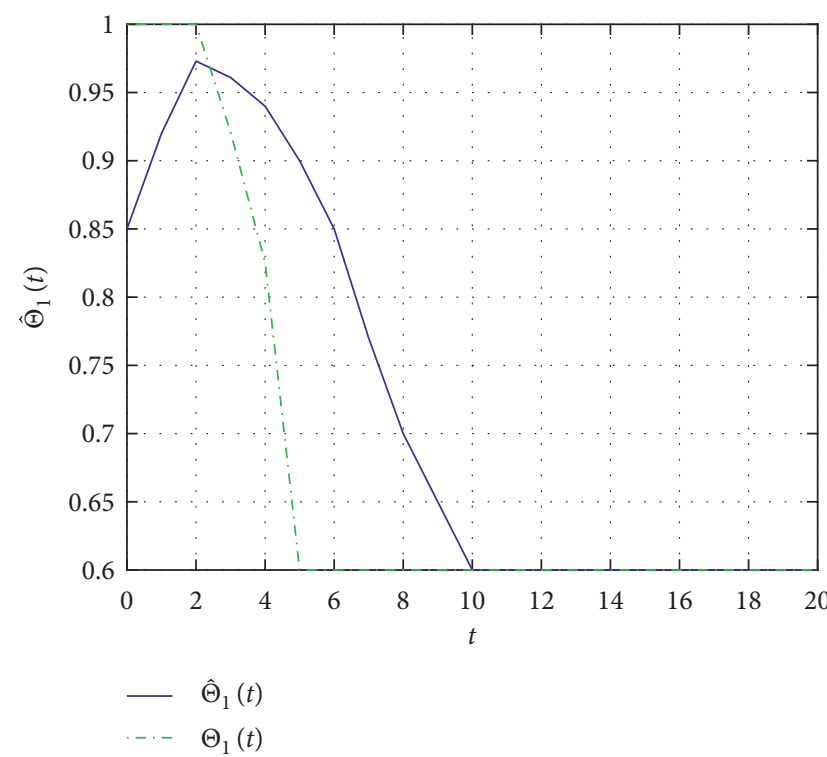

(a)

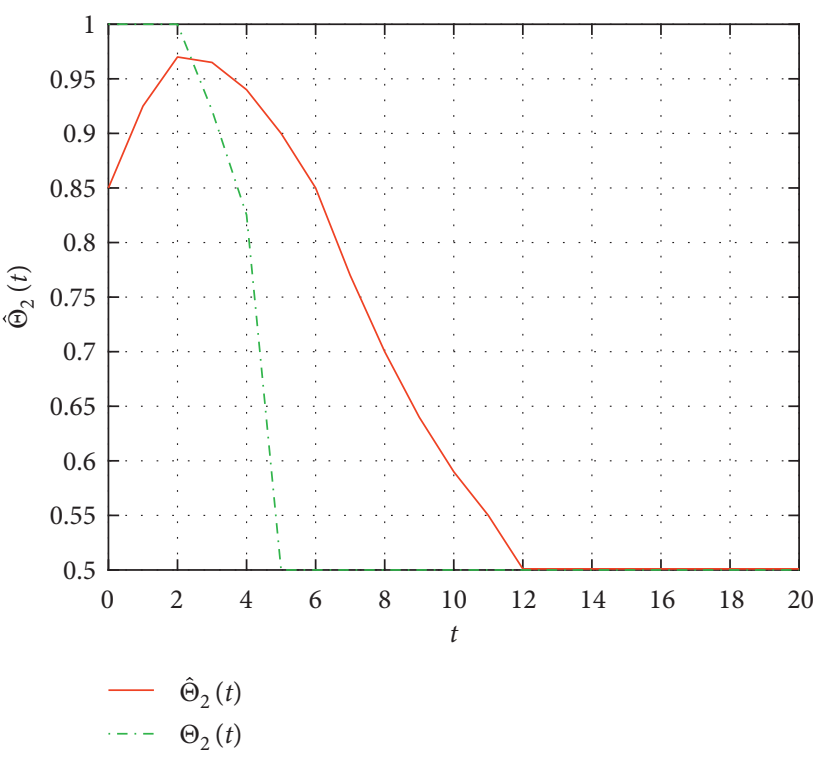

(b)

FIGURE 10: Actuator partial fault efficiency factors and the estimations.

Moreover, the state response of open-loop and closedloop of NSUSVs (25) is depicted in Figures 7 and 8, respectively. It is not difficult to find that the primary openloop of NSUSVs is unstable from Figure 7. Comparably, it can be seen from Figure 8 that the state feedback trajectory of the closed-loop of NSUSV gradually converges to the stable level despite both the partial fault and the bias fault that exist in the actuator when the proposed fault-tolerant controller of Theorem 2 is employed.

On the other hand, Figures 10 and 11 demonstrate the estimations fault efficiency coefficients of actuator partial fault and the estimations of the upper and lower bounds of actuator bias fault, respectively. More exactly, it can be known from Figure 10 that when $t<2$ seconds, the actuator 


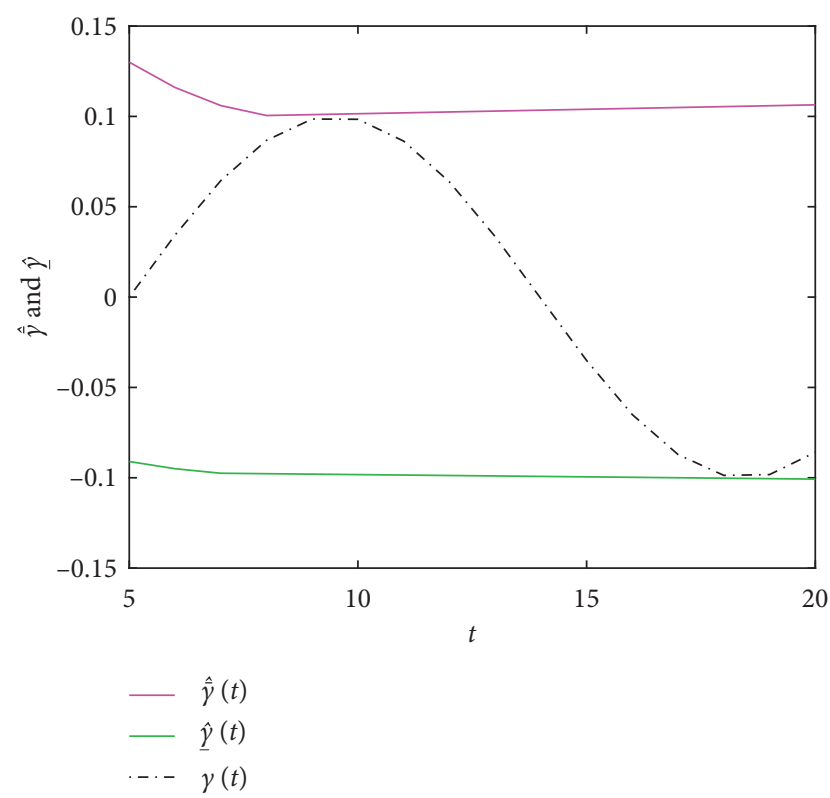

Figure 11: Actuator bias fault and estimations of its upper and lower bounds.

is functioning normally, that is, $\Theta_{1}=\Theta_{2}=1$. When $t=2$ seconds, the actuator partial fault begins to occurs, and when $t=5$ seconds, the faults efficiency factors start to reach equilibrium, that is, $\Theta_{1}=0.6$ and $\Theta_{2}=0.5$. The bias fault of actuator emerges from $t=5$ seconds, and the tracking performances of actuator bias faults have achieved an ideal performance from Figure 11.

It is worth noting that the work [44] addresses the codesign problem of a fault detection filter and controller for a networked-based USVs system subject to external disturbance and actuator fault under a conventional ETM (its limitation has been discussed above). Since the asynchronization problem, multiple types of actuator faults, and the proposed AETM are not taken into consideration in this work, the controller designed by Ma et al. cannot guarantee that our proposed NSUSVs are stable (for detail, see Figure 9); in other words, the effectiveness of the proposed adaptive event-triggered asynchronous fault-tolerant controller for NSUSVs is self-evident.

\section{Conclusion}

In this paper, an adaptive fault-tolerant controller has been designed for the NSUSVs suffering from external disturbance and actuator faults including the partial fault and bias fault. To boost the utilization ratio of network resources, a modedependent AETM is carried out. Moreover, the asynchronizations among the controlled system, adaptive fault-tolerant controller, and faulty actuator are taken into consideration, which are described by two corresponding HMMs. Under this framework, a sufficient condition is provided in order to guarantee the uniformly ultimate boundedness of the solutions by Lyapunov-Krasovskii functional. Finally, an example and a comparison are presented to demonstrate the validity and feasibility of the proposed design techniques.
In the future, we will pay close attention to the double event-triggered mechanisms, in the sensor-to-controller and controller-to-actuator channels, where both the communication resources consumption in the network and control updates can be reduced. It broadens the trade-off option between NSUSV dynamic performance and network resource utilization.

\section{Data Availability}

The data supporting this research are from previously reported studies and datasets, which have been cited. The processed data are available from reference [44] in the article.

\section{Conflicts of Interest}

The authors declare that they have no conflicts of interest.

\section{Acknowledgments}

This work was supported by the Zhejiang Provincial Natural Science Foundation of China (No.LY19F030020), National Natural Science Foundation of China (No.61733009), and NSFC-Zhejiang Joint Fund for the Integration of Industrialization and Informatization (No. U1709215).

\section{References}

[1] Y. Liu and R. Bucknall, "Path planning algorithm for unmanned surface vehicle formations in a practical maritime environment," Ocean Engineering, vol. 97, pp. 126-144, 2015.

[2] Y. Liu and R. Bucknall, "The angle guidance path planning algorithms for unmanned surface vehicle formations by using the fast marching method," Applied Ocean Research, vol. 59, pp. 327-344, 2016.

[3] L. Ma, Y. Liang, J. Chen et al., "Stabilization for damping multimachine power system with time-varying delays and sector saturating actuator," Mathematical Problems in Engineering, vol. 2016, Article ID 2958240, 13 pages, 2016.

[4] H. Ma, E. Smart, A. Ahmed, and D. Brown, "Radar imagebased positioning for USV under GPS denial environment," IEEE Transactions on Intelligent Transportation Systems, vol. 19, no. 1, pp. 72-80, 2018.

[5] Y.-L. Wang and Q.-L. Han, "Network-based heading control and rudder oscillation reduction for unmanned surface vehicles," IEEE Transactions on Control Systems Technology, vol. 25, no. 5, pp. 1609-1620, 2017.

[6] Y. Liao, Q. Jiang, T. Du, and W. Jiang, "Redefined output model-free adaptive control method and unmanned surface vehicle heading control," IEEE Journal of Oceanic Engineering, vol. 45, no. 3, pp. 714-723, 2020.

[7] Z. Peng, J. Wang, and D. Wang, "Distributed containment maneuvering of multiple marine vessels via neurodynamicsbased output feedback," IEEE Transactions on Industrial Electronics, vol. 64, no. 5, pp. 3831-3839, 2017.

[8] Z. Peng, J. Wang, and D. Wang, "Distributed maneuvering of autonomous surface vehicles based on neurodynamic optimization and fuzzy approximation," IEEE Transactions on Control Systems Technology, vol. 26, no. 3, pp. 1083-1090, 2018.

[9] H. Katayama and H. Aoki, "Straight-line trajectory tracking control for sampled-data underactuated ships," IEEE 
Transactions on Control Systems Technology, vol. 22, no. 4, pp. 1638-1645, 2014.

[10] C. Paliotta, E. Lefeber, K. Y. Pettersen, J. Pinto, M. Costa, and J. T. De Figueiredo Borges De Sousa, "Trajectory tracking and path following for underactuated marine vehicles," IEEE Transactions on Control Systems Technology, vol. 27, no. 4, pp. 1423-1437, 2019.

[11] Y. Ma, G. Zhu, and Z. Li, "Error-driven-based nonlinear feedback recursive design for adaptive $\mathrm{NN}$ trajectory tracking control of surface ships with input saturation," IEEE Intelligent Transportation Systems Magazine, vol. 11, no. 2, pp. 17-28, 2019.

[12] H. Qin, C. Li, and Y. Sun, “Adaptive neural network-based fault-tolerant trajectory-tracking control of unmanned surface vessels with input saturation and error constraints," IET Intelligent Transport Systems, vol. 14, no. 5, pp. 356-363, 2020.

[13] G. Guo, "Linear systems with medium-access constraint and Markov actuator assignment," IEEE Transactions on Circuits and Systems I: Regular Papers, vol. 57, no. 11, pp. 2999-3010, 2010.

[14] Z.-G. Wu, P. Shi, Z. Shu, H. Su, and R. Lu, "Passivity-based asynchronous control for Markov jump systems," IEEE Transactions on Automatic Control, vol. 62, no. 4, pp. 20202025, 2017.

[15] J. Song, Y. Niu, and Y. Zou, “Asynchronous sliding mode control of Markovian jump systems with time-varying delays and partly accessible mode detection probabilities," Automatica, vol. 93, pp. 33-41, 2018.

[16] F. Li, C. Du, C. Yang, and W. Gui, "Passivity-based asynchronous sliding mode control for delayed singular Markovian jump systems," IEEE Transactions on Automatic Control, vol. 63, no. 8, pp. 2715-2721, 2018.

[17] A. M. De Oliveira and O. L. V. Costa, "Mixed (H2/Ho) control of hidden Markov jump systems," International Journal of Robust and Nonlinear Control, vol. 28, no. 4, pp. 1261-1280, 2018.

[18] Y. Liu, F. Fang, J. H. Park, H. Kim, and X. Yi, “Asynchronous output feedback dissipative control of Markovian jump systems with input time delay and quantized measurements," Nonlinear Analysis: Hybrid Systems, vol. 31, pp. 109-122, 2019.

[19] A. M. De Oliveira and O. L. V. Costa, "H2-Filtering for discrete-time hidden Markov jump systems," International Journal of Control, vol. 90, no. 3, pp. 599-615, 2017.

[20] Z.-G. Wu, P. Shi, H. Su, and J. Chu, "Asynchronous," Automatica, vol. 50, no. 1, pp. 180-186, 2014.

[21] S. Dong, Z. Wu, Y. Pan et al., "Hidden-Markov-model-based asynchronous filter design of nonlinear Markov jump systems in continuous-time domain," IEEE Transactions on Cybernetics, vol. 49, no. 6, pp. 2294-2304, 2019.

[22] Y. Shen, Z. Wu, P. Shi et al., "Asynchronous filtering for Markov jump neural networks with quantized outputs," IEEE Transactions on Systems, Man, and Cybernetics: Systems, vol. 49, no. 2, pp. 433-443, 2019.

[23] H. Shen, M. Chen, Z. Wu, J. Cao, and J. H. Park, "Reliable event-triggered asynchronous extended passive control for semi-Markov jump fuzzy systems and its application," IEEE Transactions on Fuzzy Systems, vol. 28, no. 8, pp. 1708-1722, 2020.

[24] H. Yan, H. Zhang, F. Yang, X. Zhan, and C. Peng, "Eventtriggered asynchronous guaranteed cost control for Markov jump systems discrete-time neural networks with distributed delay and channel fading," IEEE Transactions on Neural Networks and Learning Systems, vol. 29, no. 8, pp. 3588-3598, 2018.
[25] Z. Gu, E. Tian, and J. Liu, “Adaptive event-triggered control of a class of nonlinear networked systems," Journal of Franklin Institute, vol. 354, pp. 3854-3871, 2017.

[26] L. Cao, B. Xiao, and M. Golestani, "Robust fixed-time attitude stabilization control of flexible spacecraft with actuator uncertainty," Nonlinear Dynamics, vol. 100, no. 3, pp. 2505-2519, 2020.

[27] J. Jiang and X. Yu, "Fault-tolerant control systems: a comparative study between active and passive approaches," $A n$ nual Reviews in Control, vol. 36, no. 1, pp. 60-72, 2012.

[28] Z. Zuo, D. W. C. Ho, and Y. Wang, "Fault tolerant control for singular systems with actuator saturation and nonlinear perturbation," Automatica, vol. 46, no. 3, pp. 569-576, 2010.

[29] H. Li, P. Shi, and D. Yao, "Adaptive sliding-mode control of Markov jump nonlinear systems with actuator faults," IEEE Transactions on Automatic Control, vol. 62, pp. 1088-1096, 2017.

[30] J. Tao, R. Lu, P. Shi, H. Su, and Z.-G. Wu, "Dissipativity-based reliable control for fuzzy Markov jump systems with actuator faults," IEEE Transactions on Cybernetics, vol. 47, no. 9, pp. 2377-2388, 2017.

[31] D. Ye, M. Chen, and H. Yang, "Distributed adaptive eventtriggered fault-tolerant consensus of multiagent systems with general linear dynamics," IEEE Transactions on Cybernetics, vol. 49, pp. 757-767, 2019.

[32] J. Li and W. Ren, "Finite-horizon Hळ fault-tolerant constrained consensus for multiagent systems with communication delays," IEEE Transactions on Cybernetics, pp. 1-11, 2019.

[33] J. Li, X. Liu, X. Ru, and X. Xu, "Disturbance rejection adaptive fault-tolerant constrained consensus for multi-agent systems with failures," IEEE Transactions on Circuits and Systems II: Express Briefs, pp. 1-11, 2020.

[34] J. Li, K. Gu, X. Liu, and X.-B. Xu, "Asynchronous adaptive fault-tolerant control for Markov jump systems with actuator failures and unknown nonlinear disturbances," Complexity, vol. 2020, Article ID 5038238, 10 pages, 2020.

[35] H. Ma and G. Yang, "Adaptive fault tolerant control of cooperative heterogeneous systems with actuator faults and unreliable interconnections," IEEE Transactions on Automatic Control, vol. 61, pp. 3240-3255, 2016.

[36] Y. Farid, V. Majd, and A. Ehsaniseresht, "Fractional-order active fault-tolerant force-position controller design for the legged robots using saturated actuator with unknown bias and gain degradation," Mechanical Systems and Signal Processing, vol. 104, pp. 465-486, 2018.

[37] Q. Shen, B. Jiang, P. Shi, and J. Zhao, "Cooperative adaptive fuzzy tracking control for networked unknown nonlinear multiagent systems with time-varying actuator faults," IEEE Transactions on Fuzzy Systems, vol. 22, no. 3, pp. 494-504, 2014.

[38] G. Chen and Y. Song, "Fault-tolerant output synchronization control of multi-vehicle systems," IET Control Theory and Applications, vol. 8, no. 8, pp. 574-584, 2014.

[39] J. Li, Y. Xu, K. Gu, L.-S. Li, and X.-B. Xu, "Mixed passive/Ho hybrid control for delayed Markovian jump system with actuator constraints and fault alarm," International Journal of Robust and Nonlinear Control, vol. 28, no. 18, pp. 6016-6037, 2018.

[40] B. Xiao, L. Cao, S. Xu et al., "Robust tracking control of robot manipulators with actuator faults and joint velocity measurement uncertainty," IEEE/ASME Transactions Mechatronics, vol. 25, no. 3, pp. 1354-1365, 2020. 
[41] X. Jin, S. Wang, J. Qin, W. X. Zheng, and Y. Kang, "Adaptive fault-tolerant consensus for a class of uncertain nonlinear second-order multi-agent systems with circuit implementation," IEEE Transactions on Circuits and Systems-I: Regular Papers, vol. 65, no. 7, pp. 2243-2255, 2018.

[42] X. Jin, S. Lv, J. Qin, and W. X. Zheng, "Auxiliary constrained control of a class of fault-tolerant systems," IEEE Transactions on Systems, Man and Cybernetics: Systems, pp. 1-12, 2019.

[43] L. Chen, M. Liu, X. Huang, S. Fu, and J. Qiu, "Adaptive fuzzy sliding mode control for network-based nonlinear systems with actuator failures," IEEE Transactions on Fuzzy Systems, vol. 26, no. 3, pp. 1311-1323, 2018.

[44] Y. Ma, Z. Nie, S. Hu, Z. Li, R. Malekian, and M. Sotelo, "Fault detection filter and controller co-design for unmanned surface vehicles under DoS attacks," IEEE Transactions on Intelligent Transportation Systems, pp. 1-13, 2020.

[45] H. Wang, P. Shi, C. Lim, and Q. Xue, "Event-triggered control for networked Markovian jump systems," International Journal of Robust and Nonlinear Control, vol. 25, no. 17, pp. 3422-3438, 2015.

[46] X. Liu, H. Zhang, T. Wu, and J. Shu, "Stochastic exponential stabilization for Markov jump neural networks with timevarying delays via adaptive event-triggered impulsive control," Complexity, vol. 2020, Article ID 3956549, 15 pages, 2020.

[47] R. Horn and C. Johnson, Matrix Analysis, Cambridge University Press, Cambridge, UK, 1987.

[48] L. Xie, "Output feedback $H \infty$ control of system with parameter uncertainty," International Journal of Control, vol. 63, pp. 741-750, 1996.

[49] H. Khalil, Nonlinear Systems, Prentice-Hall, Upper Saddle River, NJ, USA, 3rd edition, 2002. 